Portland State University

PDXScholar

5-9-1996

\title{
An Integrated Approach to Theories of Loss and Adaptation to Disability
}

Marjorie Anne White

Portland State University

Follow this and additional works at: https://pdxscholar.library.pdx.edu/open_access_etds

Part of the Counselor Education Commons, and the Education Commons Let us know how access to this document benefits you.

\section{Recommended Citation}

White, Marjorie Anne, "An Integrated Approach to Theories of Loss and Adaptation to Disability" (1996). Dissertations and Theses. Paper 5143.

https://doi.org/10.15760/etd.7019

This Thesis is brought to you for free and open access. It has been accepted for inclusion in Dissertations and Theses by an authorized administrator of PDXScholar. Please contact us if we can make this document more accessible: pdxscholar@pdx.edu. 


\section{THESIS APPROVAL}

The abstract and thesis of Marjorie Anne white for the Master of Science in Education: Counselor Education were presented May 9, 1996, and accepted by the thesis committee and the School. COMMITTEE APPROVALS :

Hanoch Livneh, Chair
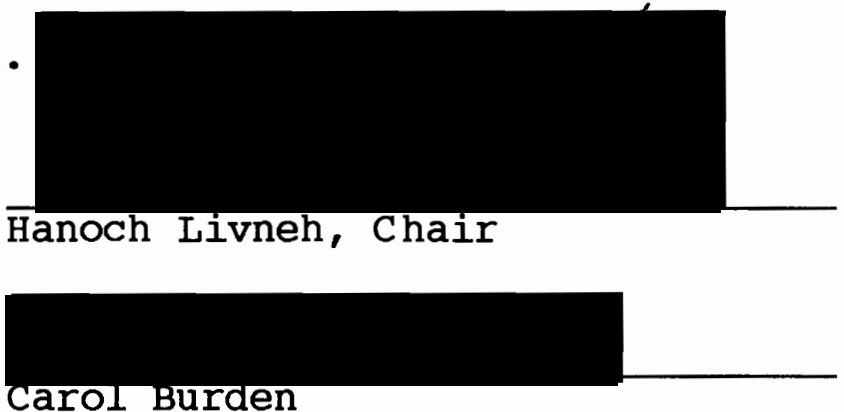

SCHOOL APPROVAL:

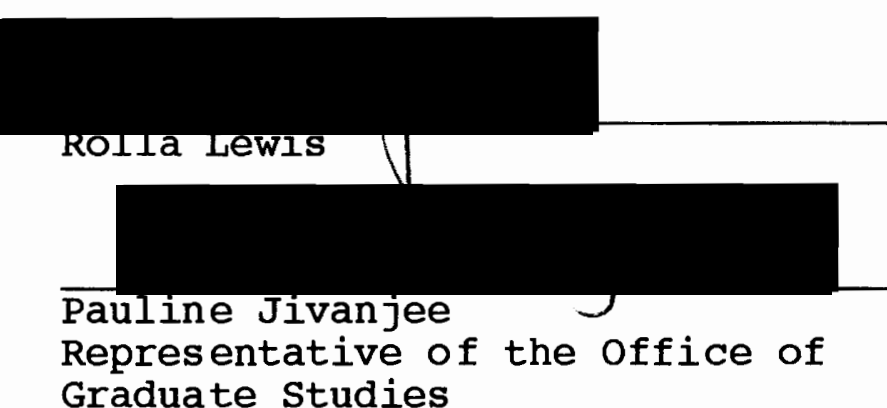
Graduate Studies

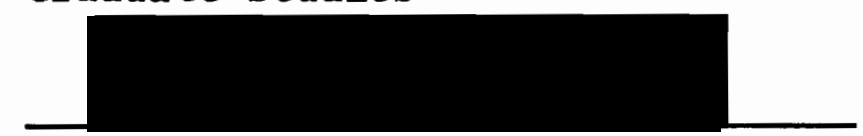

Robert B. Everhart, Dean

School of Education

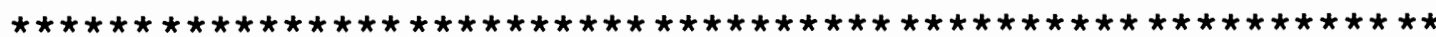
ACCEPTED FOR PORTLAND STATE UNIVERSITY BY THE LIBRARY by on 4 Qtene 1946 


\section{ABSTRACT}

An abstract of the thesis of Marjorie Anne White for the Master of Science in Education: Counselor Education presented May 9, 1996.

Title: An Integrated Approach to Theories of Loss and Adaptation to Disability.

Many theories have been proposed that attempt to explain response and adaptation to loss. However, no one theory has been shown to be universally applicable to all individuals suffering a loss. This paper presents an overview of many of these theories, paying special attention to the relationship between the theories of loss and adaptation to disability. The theories include the psychoanalytic model of loss, stage models of adaptation to loss, the value change theory of acceptance of loss, chronic sorrow, the cognitive adaptation theory, the stress and coping model, developmental theory, and the existential perspective on loss and grief. The possible influence that factors such as age, gender, culture and variables specific to disability may have on loss are also discussed. 
A new conceptualization of loss is introduced that tries to integrate many of the key ideas of these theories. This integrated approach takes into account the unique situation of each person, emphasizing the interaction between environmental and personal factors in adapting to loss. The model contends that due to the uniqueness of the individual, to the many possible combinations of environmental and psychological factors, and to the nature of the loss itself, any one or combination of loss theories could be in effect for any single loss. The intent of this thesis is to encourage the reader to become familiar with different perspectives on the subject of loss and grief to help select the method that best fits the individual situation of the person seeking counseling. 
AN INTEGRATED APPROACH TO THEORIES

OF LOSS AND ADAPTATION TO DISABILITY

by

MARJORIE ANNE WH ITE

A thesis submitted in partial fulfillment of the requirements for the degree of

\author{
MASTER OF SCIENCE \\ in \\ EDUCAT ION : \\ COUNSELOR EDUCATION
}

Portland State University

1996 


\section{ACKNOWLEDGMENTS}

I would like to thank Hanoch Livneh for all the advice, support, and encouragement he has offered to me in the past year. His knowledge of the topic of loss was a valuable resource. I am also grateful to my other committee members, Carol Burden and Rolla Lewis. Their feedback gave me the momentum to move one step further in the development of my thesis. I owe special thanks to my nephew Zach. Without the inspiration that only a 15-year old can offer, I may never have started this project. Zach's input helped me set my initial goal of writing a thesis and to eventually realize that there really was an end to the whole process. 
TABLE OF CONTENTS

PAGE

ACKNOWLEDGMENTS $\ldots \ldots \ldots \ldots \ldots \ldots \ldots \ldots \ldots \ldots \ldots \ldots \ldots \ldots \ldots$

LIST OF FIGURES $\ldots \ldots \ldots \ldots \ldots \ldots \ldots \ldots \ldots \ldots \ldots \ldots$ v

CHAPTER

I INTRODUCTION $\ldots \ldots \ldots \ldots \ldots \ldots \ldots \ldots \ldots \ldots$

II TERMINOLOGY $\ldots \ldots \ldots \ldots \ldots \ldots \ldots \ldots \ldots \ldots \ldots \ldots$

III THEORIES OF LOSS AND GRIEF $\ldots \ldots \ldots \ldots \ldots \ldots 18$

Psychoanalytic Model of Loss ......... 18

Classical Psychoanalytic Model

Contemporary Psychoanalysis

Alfred Adler

Stage Models of Adaptation ......... 35

Linear Models

Cyclical Models of Adaptation

Stage Models of Adaptation to

Disability

The Role of Denial and Depression

The Debate Concerning Stage Models

Value Change Theory of

Acceptance of Loss ............. 70

Chronic sorrow $\ldots \ldots \ldots \ldots \ldots \ldots \ldots \ldots 77$

Cognitive Adaptation Theory $\ldots \ldots \ldots \ldots 82$

The Stress and Coping Model $\ldots \ldots \ldots \ldots 85$

Developmental Theory $\ldots \ldots \ldots \ldots \ldots \ldots . .91$ 
The Existential Perspective of Loss ... 103 VARIABLES INFLUENCING RESPONSE TO LOSS $\ldots . .107$

Age ............................... 108

Infancy and Early childhood

The School-aged Child

Adolescence

Young Adulthood

Middle Age

Older Adulthood

Gender ........................... 121

Culture $\ldots \ldots \ldots \ldots \ldots \ldots \ldots \ldots \ldots \ldots \ldots$

Variables Specific to Disability ...... 134

V CHOOSING A THEORY OF LOSS .............. 140

The Attributes of a Good Theory ....... 140

Arguments for an Integrated Theory

of Loss ....................... 144

An Integrated Model of Loss .......... 151 


\section{LIST OF FIGURES}

FIGURE

PAGE

1. An integrated theory of loss ........... 153 
CHAPTER I

\section{INTRODUCTION}

Loss and grief have been topics of investigation for many years. Darwin (1872/1965) studied expressions of grief among both humans and animals. He described how sorrow is expressed by animals, young children, and adults, identifying features common to all three groups (Parkes, 1972). His work was an inspiration to both Bowlby and Parkes in the formation of their biological theories of grief. Darwin observed the initial reaction to loss in humans to be "frantic grief" - a state of excitement accompanied by wild and violent movements (Darwin, $1872 / 1965)$. Once the person realizes nothing can be done to save the lost one, he or she will fall into "a state of low spirits" or be "utterly cast down and dejected" (p. 176). Darwin believed the sufferer benefited from the urging by friends to stay active rather than succumb to silent and motionless grief. He claimed that such exertion will stimulate the heart, which in turn reacts on the brain, aiding the mind to "bear its heavy load" (p.81). Darwin analyzed numerous photographs of grieving individuals eventually identifying "grief-muscles," 
distinguished by distinctive furrows extending across the forehead ( $p .179$ ). He noted that all the facial features of the subjects were lengthened, sinking downward from their own weight - that "down in the mouth" is the same as being out of spirits. Darwin thought this process was instinctual, the brain sending messages for muscles to contract in cases of distress. He felt that although these "grief-muscles" are inherent in all mankind, they are used more by women and children. According to Darwin, the adult human can easily prevent the screaming commonly seen in infants, but will involuntarily and unconsciously perform the expressions of grief in reaction to a melancholy thought.

A number of other approaches have been influential in formulating people's assumptions about loss (Wortman \& Silver, 1987). The classical analytic model of grief is based on the work of sigmund Freud, much of it drawn from Mourning and Melancholia (Freud, 1917/1957). To Freud, the work of mourning is complete when reality predominates, demonstrating that the love object no longer exists. The attachment to the lost object is gradually withdrawn, freeing the ego once again. Bowlby is credited with developing the attachment model of grief (Bowlby, 1961, 1973, cited in Wortman \& Silver, 1987). While Freud conceptualized grief work as the relinquishment of the love 
object, Bowlby saw it as reunion. Other popular stage models were developed by Lindemann (1944), Engel (1964), Kübler-Ross (1969), and Parkes (1972). The validity of stage models has recently been challenged because the available research data suffer from methodological shortcomings (Wortman \& Silver, 1989). Wortman and Silver fear that individuals who fail to work through and experience the stages in a particular fashion will be judged to be acting abnormally or inappropriately. They suggest there are at least three different patterns of responding to loss (Wortman \& Silver, 1987). Wortman and Silver argue that universal processes of grief fail to account for the individual variability in response to loss. Loss is a certainty in life, losses often becoming more frequent as people age (Weenolsen, 1988; Wortman \& Silver, 1987). Mourning a loss is not limited to the death of a person but may be experienced in relationship to other things as well. Such a list might include loss of health, of body parts, of valued objects, of social status, of ideals, of a dream, or of future expectations (Blacher, 1970; Cassel, 1982). Every event in a person's life involves change, which in turn involves some sort of loss that the individual attempts to overcome (Weenolsen, 1988). Weenolsen (1988) claims transcendence is completed when the individual restructures life and self in a different way. 
Cassel (1982) recognized losses as being both universal and individual. They may have features that are common to all people, but at the same time contain features that can only be defined in relationship to a specific person at a specific time.

Although many stage theories were initially applied to the loss of a person, they have been expanded to include other losses in life. Parkes (1972) applied his stage theory of bereavement to the loss of a limb and the loss of a home. Kübler-Ross (1969) originally focused on dying patients but later extended her stages of acceptance to include family members and friends of the patient, hospital staff, people with disabilities, senior citizens, and to anyone having to "come to grips with any loss" (KuiblerRoss, 1974 , p. 145). Studies have been conducted to try to identify common patterns in reaction to loss in traumatically injured individuals (Marshak \& Seligman, 1993). There has not yet been agreement on either the sequence or universality of stages of adjustment in this population. Despite this lack of consensus, Marshak and Seligman (1993) see the common elements described in the mourning process as generally useful in providing a guide for understanding behavior and for offering support following loss. 
Davis (1987) believes people with disabilities experience grief differently, that using a stage model in such cases distorts the experience and limits the understanding of mourning. Mourning in individuals with permanent disabilities, chronic illnesses, degenerative conditions, and mental retardation or illness is cyclic or recurrent and requires coming to terms with a changed self. The term chronic sorrow was first used to describe the grieving experience of mothers with mentally retarded children (Olshansky, 1962). Today, this concept of recurrent grief is believed by some theorists to be a normal state, occuring in both the individual with a chronic or life-threatening condition and in family members (Hainsworth, Eakes, \& Burke, 1994).

Other theorists have offered explanations accounting for adaptation to serious life events. Wright (1983) expanded on the earlier work of Dembo, Leviton and Wright (1956). She defined "acceptance of disability" as a shift in the individual's value system to where the disability is perceived as "nondevaluating." Vash (1981) conceptualizes acknowledgement of disability on three levels: recognition of the facts, aceptance of the implications, and embracing of the experience. Once the disability is acknowledged at these three levels, there is the possibility for transcendence. With transcendence, the disability fades 
into the background. According to Vash (1981), disability is initially experienced with neutrality and later with positive regard as growth occurs.

Horowitz (1983) proposes a cyclical model of reaction to loss that is based on developmental concepts. The mind functions to maintain inner models of self and the world. Serious life events will eventually change these models but time is necessary to process the implications of the news. The process of mourning is complete when the inner models conform to the new reality. Horowitz chooses to use the word "phases" rather than "stages" when he describes this process. In her theory of cognitive adaptation, Taylor (1983) presents an adjustment process to threatening events that is centered around three themes. They include the search for meaning in the experience, the attempt to regain mastery, and an effort to restore self-esteem. Wortman and Silver (1992) found striking variability in individual reaction to loss. They hypothesize that when a major loss is experienced, the severity of its impact will largely depend on whether it can be incorporated into the individual's view of the world.

The Institute of Medicine (IOM) appointed a committee to study what factors influence the bereavement process and the impact of bereavement on general and mental health (Osterweis, Solomon, \& Green, 1984). While recognizing the 
similarities between bereavement and other stressful events and that other losses also result in grief, the report focused on reactions to death of a significant person. The committee's search resulted in several major conclusions concerning the consequences of bereavement. First, almost everyone experiences appreciable distress with bereavement. Second, this distress interferes with functioning for at least a year and can alter the survivor's lifestyle for as along as three years. Third, there is great variety in intensity in individual reaction to bereavement, and the variability is due to intertwining psychological, social, and biologic processes. Osterweis (1984) and his colleagues downplayed the traditional stage theories stating the impact of death "cannot be neatly plotted in a series of well-defined stages" (p. 283). They concluded that a) movement seldom occurs in a straight path, b) individuals vary in the speed of recovery and the frequency of moving back-and-forth between phases, and c) some people are at increased risk for illness and even death. In their analysis of articles on grief, Rodgers and Cowles (1991) concluded that grief is a highly individualized process, that it can potentially occur in response to any perceived or anticipated loss, and results in the establishment of a new reality and a new personal identity. 
The theories mentioned above represent many of the major assumptions regarding how people react to loss. Viewed on a continuum, there are people who believe it is necessary to experience the stages of grief in order to reach the acceptance of loss (Hughes, 1980; Lindemann, 1944; Parkes, 1972), those who question whether distress is inevitable (Wortman \& Silver, 1987), and those who see reaction to loss as a chronic, recurrent process (Davis, 1987; Hainsworth, Eakes, \& Burke, 1994). Many of the contemporary theoretical models have chosen to emphasize phases or processes in mourning rather than stages (Corr, 1991-1992). Chapter 3 will take a closer look at these theories and other literature on grief and loss to attempt to discover some consistency in how people adapt to and cope with loss. Special attention will be paid to the relevancy of theories of loss to disability.

Even if there was a consensus on how people cope or adapt to loss, this would only account for a selected population recovering from loss. There are many variables that could influence the process and outcome and adapting to loss, among which are age, gender, culture, and pre-loss personality attributes and coping abilities (Wortman \& Silver, 1987). Chapter 4 will cover the literature relevant to these topics that might help account for diverse reactions to loss. It will also include a 
discussion of other factors specific to disability and their relationship to loss. Chapter 5 will be an attempt to formulate an integrated approach to grief and loss that accounts for the unique situation of each individual. 
CHAPTER II

TERMINOLOGY

Despite extensive literature related to grief, there is no agreement among the authors on an empirical definition of grief (Rodgers \& Cowles, 1991). Rodgers and Cowles suggest the failure to define grief has slowed the progress in reaching a clear understanding of the concept. They note multiple conceptualizations of the concept of grief in the literature and the confusing interchange of terms such as bereavement with grief or grief process. Due to the lack of consensus, individuals studying the topic have had to rely on their own interpretations of the concept of grief which has added to the confusion. In their analysis of the literature on grief, Rodgers and Cowles (1991) did not intend to determine what grief actually is, but to identify the manner in which the experience of grief is defined by those studying the topic.

Considering the discrepancies in the definitions of grief, mourning, bereavement, and loss, there is good reason for the confusion. Trauner, the German word for mourning, can mean both the affect of grief and the demonstration of grief (Freud, 1917/1957). Mourning was 
the English word selected throughout Freud's paper on mourning and melancholia to represent grief. Freud used the term mourning to describe an internal psychological state and process (Osterweis et al., 1984). He defined mourning as the reaction to the loss of a loved one, or to more abstract losses such as ideals, freedom, or one's country (Freud, 1917/1957). Rodgers and Cowles (1991) found mourning to be a distinct concept to characterize a response to loss. It was used in reference to social expressions of grief, as well as to describe rituals that are performed as part of the response to loss.

Other theorists have used definitions that best support their assumptions about loss. Lindemann (1944) described grief as a syndrome consisting of psychological and somatic symptomatology. Engel $(1961,1964)$ conceptualizes the term as a typical response to the loss of valued objects which constitute psychological sources of gratification. These sources include whatever a person is familiar with and is a natural and expected part of his or her environment. The loss of loved ones, a pet, a job, country, status, a body part, an ideal, or pleasant memories can jeopardize one's sense of continuity and selfconfidence. Bowlby (1961) defines mourning as the psychological process that are set in motion by the loss of a loved object and that most often leads to the 
relinquishment of the object. Grief connotes the sequence of subjective states that follow loss and accompany mourning.

The term bereavement has been consistently interchanged with grief or grief process by many authors, despite evidence that the terms do not stand for the same concept (Rodgers \& Cowles, 1991). Bereavement was conceptualized as a state resulting from experiencing the death of a significant other by some and as an antecedent to grief by others (Demi \& Miles, 1986, cited in Rodgers \& Cowles, 1991). Heikkinen (1981) saw grief as a process of separating oneself from the loss enabling the person to survive, to make essential life changes, and to make way for new attachments. Bereavement is visualized as a yardstick to measure the time needed to overcome any loss. Karl (1987) also described grief as a process, one of recovery from a threatened or shattered self-concept world view. The significance of the loss determines the severity of the process and is defined by the person's own value system.

The lack of consistent definitions of the concepts of loss could mislead the reader. Weenolsen (1988) defines loss as anything that destroys some aspect of life and self, whether it be large or small. She views loss as comprised of two phases of mourning, the first of which is 
grieving. The grieving stage is often characterized by shock and numbness, followed by yearning. Peretz (1970a) uses the term grieving to label the period of convalescence in which the ties to the lost object are gradually withdrawn allowing the freeing of energies to establish new ties. This is a time of painful suffering, common reactions being weeping, wailing, failure to eat, tearing of the hair, and restless pacing.

The analysis conducted by Rodgers and Cowles (1991) revealed that grief was highly individualized. They found little agreement among authors on what constitutes a "normal" response to loss. Rodgers and Cowles discussed the wide variety of symptoms and manifestations of grief, and the factors influencing the response to grief. They felt the large number of terms used in reference to grief is partially responsible for much of the ambiguity and confusion about grief. To clarify some of the confusion, they recommended using the term bereavement in reference to the state of having sustained a loss, and mourning to describe the social or cultural practices that are a part of the response to loss.

Another problem concerning terminology is defining terms such as adjustment, acceptance, adaptation, and coping to loss (Kowalsky, 1978, Russell, 1981; Vash, 1981). Russell (1981) uses the term adjustment to explain "how and 
to what end a person deals with crises and threatened psychological functioning" (p. 330). He found many of the same faults in the literature in his search for a satisfactory definition of adjustment as Rodgers \& Cowles (1991) did in their search for a definition of grief. There are multiple definitions of adjustment possible, the variance attributable to the theoretical perspective of the author. Russell also found that terms such as adaptation, coping, mastery, and adjustment are often used by different writers to identify the same or similar forms of psychological phenomena. A third complication is that the term adjustment can be defined in either a process-oriented or in an outcome-oriented way.

The committee appointed by the IOM to study the bereavement process reported having a difficult time defining the "end of the bereavement process" (Osterweis et al., 1984, p. 52). They deliberated over such terms as recovery, adaptation, and completion, finding none of the meanings fully satisfactory. Although recovery is seen as essential in understanding outcome, it may leave the impression that grief is an illness or that those who recover are left unchanged by the loss. Adaptation and adjustment were also seen as relevant terms but were too limiting, possibly carrying the connotation of "making the best of an unpleasant situation" (p. 52). Completion was 
seen as lacking in that it suggests that there is an identifiable endpoint to bereavement after which there is no more grief. The committee eventually agreed that no one term is adequate to account for the end of bereavement, advising the use of varied terminology to describe the multiple issues associated with the outcome of bereavement. In reference to disability, Kowalsky (1978) feels adaptation and coping are the better concepts for describing a grief process that is worked through. Adjustment implies finding personal meaning in the disability, a task the person may not be able to accomplish. The client who appears well-adjusted may not have completed mourning or grief work. Learning to live with a disability and enjoying life again does not automatically imply acceptance, that is, agreement that the disability is all right.

In the early period of the rehabilitation movement, the importance of accepting of one's disability was deemed to be good and was encouraged as long as the person did not like their disability (Vash, 1981). The term acceptance grew out of favor by the late 1950s, accepting now seen as meaning settling for second-rate hopes and goals. "Adapting to" and "coping with" became the popular terms, congruent with the gradual shift of the emphasis of modification away from the person to the environment. More 
recently, the term acceptance has been used again but with a different connotation. As with any reality, if something cannot be changed, it must be accepted if survival and growth are to occur. What is not acceptable are the handicapping conditions forced on people with disabilities by conditions in an unaccomodating world or the failure of the individual to reconcile his or herself to what is and to move on.

Osterweis et al. (1984) also found discrepancies in usage of terms pertaining to loss, the same words employed by different disciplines to describe the process and its consequences. The committee came to an agreement in definitions of terms commonly associated with loss. The definitions suggested by the IOM committee on bereavement, will be used throughout this paper (Osterweis, 1984). Bereavement is defined as the "fact of loss through death" (p. 9). Bereavement reactions refers to "any psychologic, physiologic, or behavioral response to bereavement" (p. 9). The bereavement process is "an umbrella term that refers to the emergence of bereavement reactions over time" (p. 10). Grief is "the feeling (affect) and certain associated behavior, such as crying" (p. 10). The IOM committee defined the grieving process as "the changing affective state over time" (p. 10). Mourning represents "the social expressions of grief, including mourning rituals and 
associated behaviors"(p. 10) - The term adaptation will be used in reference to integrating the reality of the loss or disability to the individual's psychological and behavioral states. 
CHAPTER III

THEORIES OF LOSS AND GRIEF

Psychoanalytic Model of Loss

\section{Classical Psychoanalytic Model}

The classical psychoanalytic model of bereavement is historically viewed as one of the most influential approaches developed to explain how people react to loss (Wortman \& Silver, 1987). Psychoanalytic theorists have gone beyond the descriptive approach in trying to understand the reactions of grieving individuals (Thurer, 1985). The psychoanalytic model is largely based on Freudian theory beginning with "Mourning and Melancholia" (Osterweis et al., 1984; Zisook \& DeVaul, 1985) Freud proposes the existence of intrapsychic processes that help a person to come to terms with loss (Grzesiak \& Hicok, 1994). The pain of mourning is regarded as the inevitable companion of the persistent and insatiable yearning for the lost object (Bowlby, 1961). The work of mourning consists of the gradual withdrawal of the psychological attachment to the love object, the ego having succeeded in freeing its libido from the lost object. Memories binding the survivor to the lost object must be brought up and "hyercathected" 
or broken (Parkes, 1972). By this, Freud meant that the hypothetical psychic energy (libido) that has been bound to the lost objects must be successfully set free (cathexis). The ego becomes free and uninhibited once the work of mourning is completed (Stroebe, 1992-93).

Internal conflict arises when the individual experiences an intense yearning and an intense unwillingness to abandon the lost loved one, but at the same time is faced with the reality that the loved object no longer exists. It is a painful struggle involving painful emotions. An initial reaction may be that of denial of the loss. There may be a loss of interest in the outside world, a preoccupation with thoughts of the deceased, an inability to pursue new objects of love to replace the deceased, and a rejection of activities not associated with thoughts of the loved person. Memories and thoughts of the deceased are reviewed and the bereaved person's ties are gradually withdrawn. When the person is at last free from the attachment to the unavailable person, the grieving process is complete, and he or she regains adequate emotional energy to invest in a new object of interest (Freud, 1917/1957; Osterweis et al., 1984; Wortman \& Silver, 1987). Normal mourning is conceived as a significant departure from normal life but not as a 
pathological reaction (Teel, 1991). It will resolve over time and does not require medical intervention. Melancholia displays the same features of mourning with one exception (Freud, 1917/1957). Significant disturbances of self-esteem are evident in melancholia. Freud believed that melancholia is related to loss that has been withdrawn from the conscious. In the state of mourning, there is nothing about the loss that is unconscious. Freud argued that ambivalence in loverelationships was one of the most important determinants leading to depression following bereavement (Freud, 1917/1957; Wortman \& Silver, 1987). The conflict due to the ambivalence adds a pathological hue to mourning, often emerging in the form of self-reproaches. The mourner may blame himself or herself for the loss of the object feeling that he or she has willed it. Freud originally advanced the thought that many psychiatric illnesses are expressions of pathological mourning (Freud, 1917/1957; Wortman \& Silver, 1987).

Freud and disability. Several statements Freud made about people with physical disabilities would be considered derogatory today (Cubbage \& Thomas, 1989). He referred to people with disabilities as "misshapen, "crippled", and "miserable", equating them with beggars and poor people. Freud (1905/1953) identified psychosexual stages of 
development with the personality being formed in the first five to six years of life. Successful passage through the stages and the formation of a healthy personality require an optimal amount of gratification at each stage (Nye, 1986). Interruptions of a person's development in early life may result in the individual becoming fixated at a particular stage or regression to behavior characteristic of an earlier stage (Thurer, 1985), For example, a child who becomes disabled may be reluctant to move on to the next stage if too much gratification of his or her innate drives is received. Too little gratification can result in frustration and anxiety and may hinder future development. In either case, development may be blocked.

Freud maintained that humans exist at different levels of growth and development (English, 1971). According to Freud, at low maturational levels, people operate in accordance with basic instinctual drives involving sex and security needs. Only the fittest survive at this level. Today, humans rarely have to fight for their physical security, but instead continue to compete for "psychological superiority." According to psychoanalytic theory, people who are prejudiced toward people with disabilities are probably relatively immature individuals with unexpressed hostilities and a need to feel psychologically superior (English, 1971). Psychoanalytic 
theorists see behavior as being largely determined by unconscious factors, with learned behavior being formed in the preschool years of the child. A disability usually has an adverse effect on personality, especially when the onset occurs during early childhood. Although disability in childhood is likely to result in adults who are immature and passive-aggressive types, according to psychoanalytic theory disability occuring after school begins would probably not result in any substantial change in personality (English, 1971).

Despite the prevalence of feelings of inferiority ingrained in his writing, several of Freud's concepts add to the study of disability (Marshak \& Seligman, 1993). Although Freud focused on the loss of a loved object, Grzesiak and Hicok (1994) suggest the process can also be applied to the loss of a body part or body function. Freud (1917/1957) describes mourning as a gradual, yet sometimes stormy process. It involves an initial denial of the loss followed by acceptance of the loss over time. Depression is thought to be the result of reactions to the loss (Grzesiak \& Hicok, 1994). According to Freud, unfavorable life events do not cause neurosis, but it is a more likely the trauma brings forth latent neurotic tendencies. The individual's capacity to adapt to physical loss is affected by these conscious and unconscious psychological factors. 
If this is true, a person suffering from an accident is not depressed as a result of the accident itself, but because of what the pre-existing neurotic tendencies triggered by the accident (Cubbage \& Thomas, 1989; Grzesiak \& Hicok, $1994)$

Freud's concept of castration anxiety is often regarded as essential to the understanding of disability by the proponents of psychotherapy (Cubbage \& Thomas, 1989; Grzesiak \& Hicok, 1994). Freud used the term castration anxiety to describe any separation from the body. In this context, disability may represent a form of castration to the person with a disability or a threat of castration to the able-bodied person. Freudian theory gives multiple meanings to the term narcissism. In addition to self-love, narcissism can refer to a perversion, a mode of relating, or a developmental stage (Pulver, 1970, cited in Grzesiak \& Hicok, 1994). Narcissism is also viewed as being with self-esteem. The body is one of the sources of identity and self regard, damage to it may lead to severe emotional upheaval, loss of identity, and diminished self-worth. Physical disability can be a penetrating narcissistic injury (Grzesiak \& Hicok, 1994).

The psychology of trauma is an important consideration when analysts are working with physically disabled individuals (Grzesiak \& Hicok, 1994). Analytic theorists 
base their beliefs on Pierre Janet's work on memory systems (Ellenberger, 1970, cited in Grzesiak \& Hicok, 1994). Janet, a contemporary of Freud, hypothesized that traumatic memories are locked in a dissociated state of mind. People who experience early trauma will unconsciously go through reenactments of trauma in later life. Psychotherapists believe that trauma-caused physical disability can reactivate this earlier, dissociated, suffering state of mind.

Another psychoanalytic concept Grzesiak and Hicok (1994) claim is related to the understanding of physical disability is Freud's concept of Thanatos, the death instinct. Freud contended that it was the natural human condition to have a tendency toward violence and destructiveness (Cook, 1992). This destructive energy, the death instinct, is diverted outward in the form of an aggressive drive. The belief is that personality style and characteristic traits may put certain individuals at higher risk for disabling trauma. The death instinct can be connected to many self-destructive behaviors that may eventually culminate in physical disability.

While Freud initially formulated the concept of defense mechanisms, it was his daughter Anna who elaborated on them and on their application to bodily illness in children (Grzesiak \& Hicok, 1984). Ideally, an individual 
who is anxious will be able to identify the source of anxiety and take action to reduce the anxiety before it reaches a high level. Sometimes, temporary defenses are necessary to make it through an especially trying experience. Defense mechanisms are unrealistic intrapsychic processes that are adopted to provide relief from emotional conflict and to protect the person from anxiety. Defense mechanisms operate unconsciously and deny or distort reality. If defense mechanisms are used extensively, a person's function can be seriously affected (Corey, 1991; Nye, 1986). When physical disability results in formidable psychological demands, the ego defenses can serve as buffers preventing the ego from being overwhelmed by anxiety. Initially, the defense serves to facilitate functioning after onset of disability (Langer, 1994). As time passes, the usefulness of the defense diminishes as the person begins to integrate the emotional meaning of the loss.

Contemporary Psychoanalysis

Grzesiak and Hicok (1994) point out the relative unpopularity of psychoanalytic models of the mind with regard to physical disability. They believe this is due to the trend among rehabilitation professionals to dismiss the psychodynamic issues related to physical differences. Rehabilitation specialists usually place emphasis on the 
patient's remaining assets, while psychoanalytic therapists focus on dealing with premorbid personality and psychopathology of character. Grzesiak and Hicok (1994) claim that many rehabilitation professionals believe that the psychoanalytic approach is not appropriate for adjustment problems to disability since physical disability is not caused by intrapsychic conflict. Grzesiak and Hicok counter this with their viewpoint that individuals facing disability will often have multiple psychosocial and psychodynamic issues to resolve before they can successfully adapt to their disability. To support their premise, they cite research suggesting that intrapsychic conflict may be indirectly responsible for some cases of adventitious physical disability (Carnes, 1967; Fordyce, 1964). These studies found that some individuals with spinal cord injuries have personality factors and traits that results in behavior which puts them at higher risk for disabling trauma.

Thurer (1985) contends that criticisms against contemporary psychodynamic theory are unwarranted. They are largely based on half-truths or on misunderstandings of theoretical thinking and practice. She uses the term psychodynamic therapy in reference to the expanding array of approaches to psychotherapy derived from psychoanalysis. Psychodynamic therapy has been accused of discounting the 
present with its focus on the past, of minimizing selfresponsibility, of being overly deterministic and pessimistic, antiwomen, impractical, elitist, overly concerned with sex, and of being oblivious to conscious processes with its focus on the unconscious.

Thurer (1985) claims that classical psychoanalysis is rarely practiced in its pure form today, and that a number of theoretical modifications have taken place since the time of Freud. Baker (1985, cited in Thurer, 1985) summarizes these changes. First, emphasis is now placed on the ego rather than the id as the psychological area in which much significant psychological activities take place. Another trend is the increasing importance upon developmental factors in early life and their relationship to pathology. A third trend is the growth of populations (such as children, people with psychosis and with personality disorders) to whom psychodynamic therapy was not previously considered applicable. There has also been an increased focus on interpersonal factors. Psychotherapists have begun to take into account social and cultural aspects in their evaluation of the individual. The length of treatment has decreased, and therapists have become more active in treatment (Thurer, 1985). Psychodynamic techniques have become so modified, that many 
of them are identical to strategies used by rehabilitation counselors.

Langer (1994) sees psychotherapy as playing a vital role in easing the suffering of the person with a disability. This suffering, which is separate from physical pain, results from the internal psychic conflicts experienced with the onset of disability. Disability may present a challenge to the individual's basic assumptions about the world. Questions may arise concerning sense of self, self-esteem, shattered goals, and negative perceptions of self, any of which can cause suffering. She recommends therapeutic goals aimed at restoration or maintenance of the integrity of the person with the disability. Exploration of individual values serve to preserve the sense of self when faced with the possibility of the disruptions brought on by disability. Langer (1994) sees the losses incurred in disability as generally leading to some predictable responses with some individual variance among individuals. Some factors that may influence the experience of disability are future course of the condition, time since onset of disability, accompanying pain or physical discomfort, pre-disability affective and cognitive style, life stressors apart from disability, social and financial resources, psychodynamic issues, the meaning to others in society (cultural or social networks), 
and prior experience with, and meaning of disability and loss in general.

Shontz (1978) has speculated on the decline of psychoanalytic treatment. Two important reasons are psychoanalytic treatments are time-consuming and expensive. Also psychoanalytic practitioners are becoming too scarce to be employed in settings dealing exclusively with medical or physical conditions. Despite the decline, Shontz (1980) sees psychoanalytic theory as continuing to be a source of explanations of reaction to physical illness and disability. Among its contributions are the psychiatric labels that have been applied to normal reactions to physical illness.

Oral. Characterized by feelings of helplessness, excessive demands for attention; may regress to a secure, infantile state; may equate food, medicine, and care with love.

Compulsive. Rigid, opinionated; made anxious by hint of loss of control; feels need to know medical details about his or her medical condition.

Hysterical. Interpret illness or disability as threat to their attractiveness or potency, will constantly bid for attention, admiration, or sympathy. 
Masochistic. Long-suffering and self-sacrificing; want love and care, but expect it only in return for their own misery.

Paranoid. Feels persecuted and oppressed, finding fault with everything and everyone; guarded, querulous, and defensive; difficult to deal with.

Narcissistic. Feels powerful and all-important; smug, vain, arrogant or grandiose; quick to find fault in and lose confidence to those caring for them.

Schizoid. Reserved, remote, apparently involved in what is going on; noncompetitive, often antisocial; likely to be eccentric.

Defense mechanisms have also been used to explain reactions to disability (Hofmann, Becker, \& Gabriel, 1976, cited in Shontz, 1980). A person who uses intellectualization represses the emotional impact of his or her condition, consciously engaging in rational concern about its factual aspects. Rationalization is somewhat similar to intellectualization, but the person is less concerned about facts and more likely to adopt a fatalistic attitude or passive acceptance. Regression involves the return to a state of childhood dependency. It is considered normal in early stages of illness or injury but must be given up during convalescence. Reaction formation occurs when a person substitutes positive actions and 
beliefs for lost skills or beliefs that are too frightening to face. It is useful for fostering mastery and substitution of goals. People may respond to feelings of guilt, despair, or disgust by projecting them onto others. Physicians or nurses may be accused of incompetence to control feelings of personal responsibility for their own illness. Displacement is often considered to be a useful coping mechanism. Personal concern over one's condition is transferred to a less threatening object. A person who is acting out converts energy from anxiety into action, engaging in provocative, aggressive, or rebellious behaviors. Withdrawal is characterized by an attempt to manage stress by retreating into oneself. While it might be helpful temporarily, it is considered maladpative when used on a long-term basis. Denial is a defensive strategy commonly used at onset of disability. It involves the refusal to admit to the reality the condition even though there is clear evidence of its existence. It is considered to be maladaptive by some theorists and as a necessary step in adaptation by others (Shontz, 1980).

\section{Alfred Adler}

Adler is more accurately viewed as a peer of Freud rather than a follower, his role being that of a critic rather than a disciple (Papanek, 1967). Their common interest was neurosis and psychosis. Although Adler agreed 
with Freud on a number of premises, differences in their thinking first surfaced around 1908. Following a series of heated debates, Adler parted company with Freud in 1911. He developed his own theory of personality emphasizing social motivation and individuality, rejecting the concept of drives and instincts as the cause of pathological symptoms (Shilling, 1984). Adler called his new system Individual Psychology to draw attention to the indivisible nature of the individual (Rule, 1985). His ideas were often overshadowed by those of Freud with some people still regarding the two as similar.

Personal childhood events contributed to Adler's development of his theory of personality (Monte, 1987). Intimate contacts with death came early for Adler. When he was a youngster, he was run over in the streets on two occasions. His brother died in a bed next to his when Adler was only three. At five, he came very close to death after becoming ill with pneumonia. From then on, Adler recalls always thinking of himself as a physician. He had set a goal which could put an end to his childlike distress - his fear of death. Adler was also a chronically sick and weak child, suffering from rickets. Because of his physical condition, he was unable to compete with his older brother or peers and was forced into a position of inferiority. Adler discovered that he could gain sympathy 
and concern from the powerful people in his life as a result of organ defect (Monte, 1987).

Rule (1985) argues that many of Adler's concepts have been misunderstood or oversimplified, probably resulting from an overemphasis on his earlier writings. Adler is well known for his early concern for problems stemming from "organ inferiority" (Shontz, 1980). Adler hypothesized the existence of a basic inferiority in one region or organ which was attributable to developmental or genetic development. The person with the defective organ tries to compensate for the weakness by strengthening it. Many people interpreted this to mean that physical disability causes feelings of inferiority which are best dealt with by striving for superiority in another area of life (Marshak \& Seligman, 1993). It was once believed that such compensation is a positive goal of good rehabilitative practice. In reality, Adler was never able to provide support for his theory and turned his interests to other areas (Shontz, 1980),

Wright (1983) argues that Adler's doctrine can mistakenly lead to the expectation there is a large number of neurotic and psychotic tendencies in people with disabilities, especially as related to feelings of inferiority. McDaniel (1969) voiced the opinion that Adler's theory has been greatly misused and distorted in 
rehabilitation work. He sees Alder's contribution to the psychology of disability as being the promotion of the concepts of the uniqueness of the individual's development and behavior, and stressing the goal-directedness of behavior. McDaniel (1969) does not find fault with the theory but with its application. He claims that concepts such as compensation and inferiority have been so indiscriminately applied to the point that they might qualify as "laws of disability", characteristic only of people with physical imperfections and who are neurotic. Rule (1985) describes a shift in Adler's thinking, resulting in a improved way of understanding human nature. Adler switched from an organic to a sociopsychological explanation of behavior, refined his concepts of inferiority and compensation, placed increased emphasis on the creativity of the individual, and an increased focus on the personal attitude toward disability and the goals to which a disability can be directed. Adler's concept of lifestyle is his most useful contribution to looking at adapting to disability (Rule, 1985). Lifestyle is each person's unique, self-consistent, and relatively constant way of meeting his or her world (Ansbacher, 1980). Lifestyle is created by the individual within the limits of hereditary and environment. For some this style of living is broad, forming a foundation for meeting most of life's 
problems. Those who have a narrow lifestyle may be only able to deal with specific situations (Shilling, 1984). Lifestyle is largely formed by age five, the individual having created his or her own convictions about who he or she is, what he or she must become, and what is to be expected from life. Subsequent experiences are fitted into the lifestyle rather than forming it. The creative interpretation given to childhood experiences and the meaning found in them results in mature freedom of choice and self-determination in later life (Papanek, 1967). Lifestyle patterns can be helpful in attempting to understand the meaning of a congenital or acquired disability within the context of a person's unique expectations of self, of others and of life (Rule, 1985). In the case where the individual is disabled since childhood, the disability is likely to be a factor in the formation of the lifestyle. When disability occurs later in life, it is more likely the individual's lifestyle will influence the adjustment to the disability and outlook on the situation.

Stage Models of Adaptation

\section{Linear Models}

A number of theorists have offered models of adaptation to loss which involve phases or stages (Bowlby, 
1961; Engel, 1961; Kübler-Ross, 1969; Lindemann, 1944; Parkes, 1972). In the strictest sense, a stage model suggests that grief is linear and time bound, successful completion of grief work likened with resolution of grief (Teel, 1991). More recently, most advocates of stage theories have recognized that progression through the stages is not necessarily linear and moving toward a particular end point (Davis, 1987). Cyclical models of loss propose stages or phases that may shift or reoccur but will usually diminish in intensity with each repetition (Falek \& Britton, 1974; Fink, 1967; Horowitz, 1983; Shontz, 1980). Other theorists have presented stage models that are specific to adjustment to disability (Cohn-Kerr, 1961; Vash, 1981).

Lindemann's (1944) supposition of acute grief is based on 101 psychiatric interviews with bereaved patients. Some had lost a relative during treatment, others were relatives of those who died in the hospital. Bereaved disaster victims of the Coconut Grove Fire and relatives of members of the armed forces were also included in the study. Lindemann (1944) viewed grief as a definite syndrome with psychological and somatic symptomatology. He identified characteristics of normal grief that were "remarkably uniform" in the bereaved subjects (p. 141). This includes waves of discomfort consisting of somatic distress, a 
feeling of tightness in the throat, choking with shortness of breath, sighing, and an empty feeling in the abdomen. There is often a slight sense of unreality, an increased emotional distancing from others, and an intense preoccupation with the image of the deceased. Guilt is another reaction, the bereaved reliving the time before the death for indicators that he or she failed to do right by the loved one. There is often a loss of warmth in relationships with other people, marked by irritability and anger. Lindemann (1944) also noted restlessness, with the inability to sit still, moving about in an aimless fashion, constantly searching for something to do.

Lindemann (1944) describes grief work as the emancipation of bondage to the deceased, readjustment to the environment, and the formation of new relationships. Duration of grief is related to the success with which a person proceeds through this process. A major obstacle is that many people try to avoid the intense distress associated with the grief process and the expression of emotion necessary for it. Lindemann identified morbid pictures of grief, including delayed reaction, overreaction, under-reaction, and apparent absence of grief. With treatment, these conditions can be transformed into a normal grief reaction and resolution. 
Engel (1961, 1964) offered further support for the stage theory of adaptation to loss. He defines grief as the characteristic reaction to the loss of a valued object. Engel (1964) compared the experience of loss to a wound, the subsequent psychological responses to the process of healing. He chose to use the term "uncomplicated" rather than normal grief since any type of grief is a departure from the state of health and well-being. Uncomplicated grief is normal only in the sense that it is common, usual, and follows a predictable course. It is modified primarily by the abruptness of the loss, the nature of the preparation, and the significance of the lost object.

Usually, the first response is shock and disbelief. The person attempts to deny the loss and to protect himself or herself against the reality of what has occurred. This phase may last anywhere from a few minutes to hours or even days. The next stage is one of developing awareness of the loss and is often felt as a painful emptiness in the chest. It is marked by crying, anger, guilt, helplessness or hopelessness, sleep disturbance, a feeling of emptiness, decreased interest in usual activities and interpersonal relationships, and sometimes somatic symptoms of pain or other discomfort. The third stage is restitution, which involves the work of mourning. This includes the various rituals such as gatherings, the funeral, and individual 
religious and spiritual beliefs which help to initiate the recovery process. The stage of resolving the loss consists of a number of steps which progress haltingly and intermittently. The mourner's thoughts are almost entirely consumed with thoughts of the loved one, first with more emphasis on personal aspects of the loss and later on the person who died. The mourner is not yet able to accept a new love object to replace the lost person (Engel, 1961, $1964)$.

The final stage of idealization requires that all negative and hostile feelings toward the lost person be repressed. This repression may result in guilt, remorse, or even fearful feelings. There may be regret for past actions, or for inconsiderate or unkind behavior directed toward the deceased person. The bereaved individual may feel responsible for the person's death and even fear that the dead person might come back to haunt or retaliate for his or her part in the death. He or she focuses on the positive aspects of the lost relationship permitting periodic reliving of the gratification associated with the past life with the deceased. As the idealization of the dead person proceeds, the mourner consciously and unconsciously begins to identify with the deceased, taking on admired qualities and attributes of the person. Gradually, as the preoccupation with the dead person 
decreases, the feelings of sadness and the guilt associated with the ambivalent memories of the deceased also become less intense. The mourner begins to renew his or her interest in people and things not so directly concerned with the loss and with mourning (Engel, 1964).

The work of uncomplicated mourning typically takes a year or more to complete. Engel (1964) claimed successful grieving follows these predicable steps which make it possible to determine whether healing is actually taking place. He cautioned against trying to accelerate the process of grieving, of using unsound interventions, and stressed the importance of providing optimal conditions for healing.

Bowlby is credited with the development of the attachment model of grief (Wortman \& Silver, 1987, 1992). He relied heavily on psychodynamic theory, on developmental theory of children's reactions to separation, and on research looking at the mourning behavior of animals. Although trained in psychiatry and psychoanalysis, Bowlby felt very strongly that psychoanalysis put far too much emphasis on the child's fantasy and far too little on actual events (Bretherton, 1991, 1992). He headed a research team at the London Child Guidance Clinic choosing to focus on mother-child separation, separation being a clear-cut and undeniable event. 
Influenced by the work of Darwin (1872/1965) and Lorenz's (1935) account of imprinting in geese, Bowlby maintained that mourning is an instinctual process occuring in human infants and adults and in lower species (Bowlby, 1963; Bretherton, 1992). He found there was a common urge to recover a lost object evidenced by crying, searching, and angry protest directed towards the object (Parkes, 1970a). Bowlby (1961) advanced the hypothesis that once a child has formed a bond to a mother-figure, usually occuring by the middle of the first year, the break in the attachment leads to separation anxiety and. This sets in motion the process of mourning. Separation anxiety is experienced when attachment behavior is activated but cannot be shut off or terminated, the attachment figure continuing to be unavailable (Bretherton, 1991).

Bowlby (1961) believed that grief is a phasic process, the transitions from one phase to another seldom distinct and features from one phase often persist into the next (Parkes, 1970b). He originally proposed three main phases of mourning (Bowlby, 1961). The first phase is the urge to recover the lost object, during which yearning and an angry effort to recover the lost object predominate. Two of the main features of this phase are weeping and anger. As the mourning process proceeds normally, there is less focus on the lost object and a decrease in efforts to recover it. 
The second phase of mourning is intensely painful and alarming. In this phase of disorganization, despair sets in and behavior becomes disorganized. This disorganized behavior is similar to that described by Lindemann (1944). This includes restlessness, inability to sit still, moving about in an aimless manner and a painful lack of capacity to initiate and maintain organized patterns of behavior. Bowlby believes the depressive phase of mourning is a result of the disorganization of behavior patterns following loss. Like separation anxiety, depression is considered normal in that it is an inescapable aspect of life. Each time the individual is bereaved or relinquishes a major goal, he or she must accept the destruction of a part of his or her personality before reorganizing it toward a new goal (Bowlby, 1961, 1963). The third phase is a period of reorganization of behavior. In this phase, individuals are able to break down their attachment to the lost loved one and begin to establish new ties to others (Wortman \& Silver, 1987, 1992). There is a gradual return to former interests. Parkes (1970a) later convinced Bowlby that an additional phase should precede his first phase. This initial phase of numbness during which the awareness of loss is partially disregarded.

Mourning becomes pathological when the bereaved individual continues as if the lost object were still 
recoverable, remaining preoccupied with thoughts of it and actions directed toward it (Bowlby, 1963). This is an unconscious effort, insulating the individual from change. Bowlby $(1961,1963)$ suggested that mourning responses commonly seen in infancy and early childhood bear many of the hallmarks of pathological mourning in the adult. He contended that unsatisfactory responses to loss in the early years may take a course unfavorable to personality development, the adult responding to subsequent loss in a similar way.

Parkes is well known for his research on bereavement, focusing principally on grief in adult life (Bretherton, 1991). He joined Bowlby's research team at Tavistock in 1962 at which time he was able to visit widows to help chart a course of normal grief. Bowlby and Parkes worked in close collaboration, sharing their ideas on grief (Parkes, 1972). Parkes views grief as a process rather than a state. It is not a set of symptoms starting after loss that gradually fade away, but rather a succession of clinical pictures which blend into and replace one another. Grief is a complex and time-consuming process in which an individual changes his or her view of the world, a process of making psychologically real an external event which is not wanted and for which coping plans do not exist (Parkes, 1970b) . $\chi^{\prime}$ 
Parkes (1972) presents several features he believes to be major aspects of many bereavement reactions. The first is a process of realization in which the individual moves from denial or avoidance of recognition of the loss towards acceptance. A second reaction is one of alarm, characterized by anxiety, restlessness, and the physiological accompaniments of fear. The third feature is an urge to search for and to find the lost person in some form. This is followed by anger and guilt which includes outbursts targeted at those who urge the bereaved towards a premature acceptance of his or her loss. Feelings of internal loss or mutilation define the fifth feature. Another defining characteristic is the identification phenomenon which consists of the adoption of traits, mannerisms, or symptoms of the lost person. Parkes also lists pathological variants of grief which may be excessive, prolonged, inhibited or distorted reactions to loss. He feels these reactions to bereavement are found in a similar form in other types of loss. Parkes sees no clear end to grief (Parkes, 1985). The past is not forgotten, but rather the bereaved gradually discover which assumptions are still relevant and enriching in their new lives and which must be given up.

Elizabeth Kübler-Ross (1969) is one of the most wellknown and controversial stage theorists. Parkes met with 
her when she was in the process of gathering information for her book, On Death and Dying (Bretherton, 1992). Her stage-based model, which is an attempt to understand the coping process of dying, was also influenced by the work of Bowlby. It has since been widely used to explain the process of grief. Kübler-Ross (1969) based her conclusions on interviews with dying patients covering a two and a half year period. Her goal, then and now, is to educate healthcare professionals and clergy to become more familiar with the needs, concerns, fears, and anxieties experienced by individuals (and their families) as they face the end of their lives (Kuibler-Ross, 1987). According to Kuibler-Ross (1969), the stages are a) denial and isolation, b) anger, c) bargaining, d) depression, and e) acceptance. Kübler-Ross (1969) describes the first reaction to the news of having a terminal illness as a temporary state of shock. After this initial feeling of numbness begins to disappear, the individual enters the first stage of the dying process - denial and isolation. Denial is usually temporary and soon evolves into partial acceptance. When denial can no longer be maintained, it is replaced by anger, the second stage of dying. The person may experience rage, anger, and resentment. The anger is displaced in all directions, often directed toward family and staff. The third stage is bargaining. Bargaining is 
an attempt to enter into some sort of agreement to postpone the inevitable from happening. The wish is almost always for an extension of life, followed by the desire for a few days without pain. Bargaining is helpful to the individual, but only for short periods of time.

The person's numbness, denial, anger and rage are soon replaced by a sense of great loss. This leads to the fourth stage of depression. Depression may occur as a result of a past loss or from impending losses. Depression is a tool to prepare for the impending loss of all love objects, allowing for the state of acceptance. The final stage is acceptance. If the individual has been able to work through the previous stages, there will come a point at which at which he or she in no longer depressed nor angry. It a state that is almost void of feelings; the struggle over. Kübler-Ross (1969) describes the different stages as lasting for different periods of time. Rather than replacing each other, they exist at times side by side and perhaps sometimes overlapping. The common element to all the stages is hope, maintained in some form to the last moment. The individual is nourished by hope in especially difficult times. Kübler-Ross $(1974,1987)$ expanded her model to include other forms of loss besides critical illness. This includes losses experienced by friends and relatives after the patient dies, by individuals facing a 
serious handicap, by senior citizens after moving to a nursing home, by couples going through a divorce, and by families whose houses burned down.

Karl (1987) claims that Kuibler-Ross' theory is limited and potentially misleading. He believes a broader conception of the phases of grief is needed, one is which grief is presented as an adaptive process. According to Karl, grief is a complex life experience, one that cannot be neatly fit into a systematic package. It is a process of recovery from an event that has jeopardized and/or destroyed a person's self-concept and world view. The severity of the grief process is determined by the significance of the loss. The symptoms are expressed physically, psychologically, socially, and spiritually as the whole being grieves. The experience of loss throws everything out of balance. How a person expresses and reacts to grief is culturally biased and based on the unique experiences of the individual. Shock is the first reaction to the loss of a loved object. It occurs immediately and may continue for weeks. The mind is reacting to information which is threatening to its existence. Denial may take place, a disbelief that something has occurred that was previously considered unreal. Physical symptoms may include fainting, hyperventilation, screaming, weak knees, and shaking. 
Coping is the second phase of the grief process. Karl (1987) describes this phase as one of intense physical, psychological, and spiritual manifestations. The physical body may exhibit symptoms like fatigue, insomnia, weight gain or loss, hyperactivity, and reoccurring personal health issues. The person may show emotions ranging from anger to guilt, from depression to mania. The individual may become intensely interested in his or her relationships or become intensely disenchanted with them. Coping is a phase in which the person is beginning to assimilate the loss, trying both to survive and gain some equilibrium. There are attempts to repress the reality and attempts to deal with the new reality and to regain wellness. Karl believes the most accurate measure to assess the person's progress in the grieving process is know the dynamics practiced by the individual in the past. The coping phase is characterized by an attempt to bring all systems back into a state of balance and typically lasts until the end of the first year after the loss.

The final stage is one of adaptation rather than one of acceptance. The person has completed the process of rebuilding his or her world-view, one which incorporates the memories of the lost object. Karl (1987) estimates this phase to last another year. He differentiates between two types of unresolved grief, one in which the person has 
become fixed in a phase and another in which the individual is slowly moving through the process.

Schneider believes his model of loss to be unique because it is generally more applicable to life circumstances and presents growth as a potential outcome of grief (Frears \& Schneider, 1981). It is wholistic in that it demonstrates how grief affects multiple aspects of the individual including biological, emotional, behavioral, intellectual, attitudinal, and spiritual. He proposes a six phase process of resolution of grief. The first phase is initial awareness at which time a definable loss becomes a reality. The individual may experience shock, loss of balance, and be more likely to become ill. The second phase, strategies to overcome, is characterized by periods of holding on and letting go.

The third phase of grief is awareness of loss. In this phase, the person intensely explores the extent of the loss and its immediate and current implications. He or she may exhibit symptoms such as loneliness, helplessness, and exhaustion. The functions of the completions phase of grief are healing, acceptance, and resolution. It serves to free the person from the energy invested in the loss. The function of the sixth stage, empowering of self, is to heighten the individual's sense of personal power in areas that were out of balance before the loss occurred. The 
final stage, transcending the loss, enhances the individual's capacity to move beyond grief - to new commitments, balance, and wholeness in life (Frears \& Schneider, 1981).

\section{Cyclical Models of Adaptation}

Falek and Britton (1974) describe four clearly identifiable response patterns to stressful situations. They claim the process is a universal reaction to any change great enough to produce stress in the organism and occurs throughout the life cycle in response to minor as well as major traumas. A successfully functioning individual must maintain carefully balanced psychological and physiological systems. A disturbance in one of the systems creates disturbance in the other resulting in stress for the total organism. Stress triggers mechanisms intended to reestablish the steady state.

The first stage, shock and denial, is an attempt for the individual to maintain the status quo. The denial mechanisms reduce the impact of the trauma, preparing the individual for cognitive acceptance of the event. The denial period is usually short-lived or may be absent in situations where the onset of the trauma is gradual. Since the person does not acknowledge the stress, there is little motivation to commence behavioral changes necessary to 
adjust to the new reality. Anxiety, the second stage of the coping sequence, is an attempt to deal with the new situation on an intellectual level. The individual is now aware of the implications of the trauma but has not yet been able to adjust to the new reality. The result is a realistic fear, reactions ranging from generalized nervousness, over-activity, irritability, headaches, fatigue, troubled sleep, changes in appetite to somatic complaints. The stressful event has been accepted on a cognitive level but has not been experienced psychologically on an emotional level. In an attempt to relieve anxiety, the individual strives for adjustment on an intellectual level directed toward reestablishing equilibrium.

The third stage is anger and/or guilt. The individual becomes bitter and hostile after attempts at readjustment result in failure. Anger may be directed to those around the individual or directed inwardly resulting in guilt. The person is trying to resolve his or her conflict by searching for a responsible agent. As the attempts to solve the problem cognitively repeatedly fail, the individual is faced with the emotional burden of the trauma. The stage of depression follows with symptoms of sadness, withdrawal, and at times a lack of interest in usual activities. As time passes, the individual begins to 
recognize that old patterns will not work and starts trying new behaviors to adjust to the current situation. Unlike previous attempts, these new behaviors come from both an emotional and cognitive realization of the stress. As the person tries out these new behaviors, there may be a return to an earlier phase, the entire coping sequence may be repeated, or there may be fluctuation between two phases. The sequence will be repeated with diminishing intensity, establishing a new psychological equilibrium that has been gradually incorporated both on an intellectual and emotional level. Falek and Britton (1974) see these basic responses as occuring in situations of the death or loss of a loved one, severe physical injury or illness, and environmental stress.

Fink (1967) based his model of adjustment to crisis on the belief that success means not only overcoming depression and accepting loss, but produces positive psychological growth (Shontz, 1980). It is a four stage process of recurrent approach-avoidance cycles, with the person successively coping with and retreating from the problems associated with the crisis. The cycles usually diminish in both frequency and intensity with time. The shock stage is a period of intense encounter with the crisis. It occurs when the individual first comprehends the implications of the crisis and lasts a relatively short 
period of time. The second stage, defensive retreat, or denial, is a neutralizing response involving cognitive restructuring that makes the situation seem to be less dangerous or threatening than it actually is. Fink considers denial to be a necessary step, only being maladaptive if it interferes with ultimate growth (Shontz, $1973,1980)$.

The third stage of acknowledgement is a cyclical series of substages in which realization alternates with defensive retreat. Accommodation to the altered situation takes place as defensive retreat is gradually given up. Acknowledgement eventually fades into the final stage of adaptation. The four phase cycle occurs many times before adaptation is complete. In the early period of adaptation, when there is strong emotional involvement, an entire cycle may take place in a matter of a few minutes or hours. Later on in the process, the progression through the stages may be so slow and weak that it may be almost unnoticeable. Fink contends that all stages must be passed through. Upon successful completion, the individual achieves a level of psychological achievement exceeding that which existed before the crisis (Shontz, 1973, 1980).

Shontz (1980) presents a modified version of Fink's theory of adaptation, the models sharing two basic assumptions. The first one is that normal adaptation is a 
developmental sequence, and secondly, that success brings about growth and an increase in psychological maturity. Shontz's first stage is shock which is marked by feelings of detachment (Wortman \& Silver, 1987). The encounter stage is characterized by feelings of helplessness, disorganization, and panic. The individual may experience reality as overwhelming and demonstrate a type of avoidance termed retreat. The cyclical pattern involves a continual shifting between encounter and retreat. Anxiety, frustration, and depression may reoccur each time the person begins to face reality. Shontz believes these feelings are necessary for psychological growth to occur. The cycles happen less often as the adaptation process proceeds and is completed.

Horowitz (1983) has developed another cyclic model of reaction to loss. The stages are: outcry, denial, intrusiveness, working through, and completion. There is a phasic tendency with an initial period of outcry followed by either denial or intrusive states. Denial is frequently characterized by numbness and intrusion with uninvited thoughts of worthlessness and uncontrolled pangs of shame. Intrusive experiences often alternate with denial or avoidance states. When the period of intrusion becomes too painful, the individual reverts to the denial phase. In the working through period the frequency and intensity of 
these states is reduced The stage of completion occurs when a relative baseline is reached. Some pathological responses to stress are being overwhelmed by the event, panic, resorting to suicide or drugs, flooded states, frozen states, psychosomatic responses, and an inability to act or love.

Horowitz (1983) has identified common responses after loss or injury that frequently take an intrusive form in conscious experience. Awareness of these states helps the individual know what to expect and how to assist his or her responses when they do occur. The common themes are fear of repetition, fear of merger with victims, shame and rage over vulnerability, anger at the source, anger at those exempted, fear of loss of control of aggressive impulses, guilt or shame over aggressive impulses, guilt or shame over surviving, and sadness over loss.

Horowitz (1983) offers a theory that he believes answers why the compulsive repetition, the phasic states of intrusion and denial, the differences in individual response to stressful events, and resolution occur in response to loss. He combines ideas from the work of Piaget (1937/1954) and Erikson (1958) in creating his explanation. Horowitz (1983) believes that the mind operates to maintain inner models of self and the world. Negative stress results from the experience of loss or 
injury, psychological or material, real or imagined. A serious, stress-producing life event threatens the existing model. When action fails to alter the situation, then inner models or schemata must be revised to conform to the new reality.

Each person has more than one model of self-image and role relationship which are developed in childhood and change throughout life. Even though early schemata have been revised, they are also retained in their earlier form and can be reactivated at later stages. A serious life event can alter the hierarchical order of existing schemata. A shift in dominant models of self and others may occur as a part of the internalization of a stressful life event. Horowitz (1983) offers the example of a person who experiences the loss of an arm, or who is fired from a job and subsequently shifts from a competent self-image to an already existing, but dormant one, as worthless and defective. The unique response of individuals can be partially accounted for by how their developmental history impacts personal meanings of an event and how current life tasks and the environment are affected by the event (Horowitz, 1983).

According to Horowitz (1983), news of and immediate responses to serious life events are stored in active memory. In the active memory there are tense and frequent 
reoccurrence of the information connected with the event. with each repetition, as comparisons are reiterated, emotional activation increases. When other tasks take precedence, or if emotional responses such as fear, guilt, anger, or sorrow become a threat, controls come into action. Controls moderate the flow of information and reduce emotional response (Horowitz, 1983).

Optimally, controls serve to slow down the recognition process, providing tolerable amounts of new information and emotional responses. Too many controls change the state of the person to denial, and may prevent complete processing of the event. If controls fail, there are excessive levels of emotion, flooding, and retraumatization which can lead to intrusive states. Completion tendency takes place as the mind eventually processes the new information and conforms to the new reality. At this time, the new information is no longer stored in the active memory, bringing to an end the tendency for repeated representations of the event. The new information is now a part of long-term memory models and of revised inner schemata (Horowitz, 1983).

Stage Models of Adjustment to Disability

A major contribution the stage model approach has made to theories of adjustment to disability is the suggestion 
that the process moves from negative, rejecting responses to more positive and accepting responses (Russell, 1981). Cohn-Kerr's (1961) stage model of adjustment to disability is a process of regaining or establishing a normative adjustment under nonnormative condition (Vash, 1981). The five stages are points on a continuum rather than distinct states and describe common, but not inevitable phases.

In the first stage of shock, the person has not yet fully comprehended the reality of the disability, consequently showing little anxiety. Psychologically, the individual is an able-bodied person working toward the same goals he or she did as a healthy person. The stage of expectancy of recovery usually occurs when the person realizes that something is truly wrong. It is featured by preoccupation with the disabling condition - the only thing that matters is to get well. There is little motivation for learning to live with the disability since the person expects to recover. The person views the disability as a barrier, preventing access to everything that he or she believes to be worthwhile in life. As the individual begins to realize that the disability may be permanent, he or she shifts to the stage of mourning. Some people may remain at this stage permanently, resigning themselves to their fates. They are likely to consider themselves worthless and inadequate (Cohn-Kerr, 1961; Vash, 1981). 
Most individuals begin to realize that the disability will not stand in the way of meeting more basic goals and move on to the next stage of defense. Efforts are made to cope with the disability along with increased motivation to learn to function as a person with a disability. Although the individual still sees the disability as a barrier, it is believed to be one that can be fought and conquered. The defensive stage is considered neurotic if the person uses defense mechanisms to deny that barriers exist. In the adjustment stage, the individual no longer views his or her disability as a barrier, but as merely one of his or her many characteristics. The person has discovered satisfactory ways to satisfy his or her needs (Cohn-Kerr, 1961; Vash, 1981).

Vash (1981) has modified the work of Cohn-Kerr in the development of her model of adapting to permanent disability. She has condensed the five stages into two levels of acknowledgement of disability and added another level that surpasses the resumption of normalcy. Vash explains this last level as being a development into higher consciousness which is brought about, in part, by experience with disability. Level I is recognition of the facts, where the disability is viewed as a tragedy. The person comes to an understanding of the nature and limitations of the disability, that it may be permanent, 
and that social stigmatization is likely. Before this level is reached, there is a time when the individual fails to recognize even the facts, denial and acknowledgment alternating in and outside consciousness.

At Level II, acceptance of the implications, disability is viewed as an inconvenience that can be mastered. The realities of the disabling condition are acknowledged without a sense of loss and without recrimination. The individual is able to integrate the facts and implications of the disability into a chosen lifestyle. The degree of progression into this level depends on how much the person accepts, without bitterness, the circumstances life presents as opposed to lapses into periods of disappointment, rage, despondency, self-hatred, and other emotions associated with the realities and implications of disability. The third level, embracing the experience, involves taking in the disability as a positively valued experience. Disability is viewed as a growth catalyst, connected to an appreciation to what the disability has been and can be in the future. According to Vash (1981), not everybody reaches this level, the number who do being comparable to people who reach higher levels of consciousness, regardless of their experience with disability. 
Vash (1981) proposes a further state that can be reached only once the disability is acknowledged at all three levels - transcending disability. This means rising above or beyond limits imposed by such conditions as the disability itself, societal expectations, or emotional reactions to either or both. Transcending disability requires that the individual free himself or herself from the mind-body trap. The disability fades into the background only rarely coming to the forefront of consciousness.

The Role of Denial and Depression

Smith (1975) equates an inability to grieve with depression. He contends that the individual unconsciously hopes to be magically rewarded for the suffering by a restoration of the lost object. Resolution is helping the depressed person to mourn. This means helping him or her know what has been lost and discover that real hope rests in acknowledgement rather than denying loss. In identifying clinically significant differences between grief and depression, Schneider (1980) defines grief as a normal reaction to a major loss. Grief is characterized by sadness, loneliness pangs, exhaustion, and is generally self-limiting. Depression is more often viewed as a clinical syndrome identifiable by negativism, helplessness, 
lowered mood, and reduced self-esteem. The grieving person is more apt to respond to gestures of warmth and reassurance, the depressed person to pressure, promises, and urging (Peretz, 1970b). Peretz describes the severely depressed individual as unresponsive to most stimuli, "huddled and downcast in gloom" (p. 29).

Hughes (1980) views grief as representing both a return to a previous state of affairs and as a process of recovery. He claims that a person can reach the stage of acceptance of loss only after experiencing the stages of grief. The process includes shock, denial, anxiety, anger and/or guilt, and depression. The person must endure and overcome each stage before "coming to a full acceptance of reality and beginning to reconstruct his/her life" (p. 253). His opinion is similar to that held by the majority of traditional stage theorists. It is widely held that distress and depression are a part of the grieving process and their absence is a sign of pathology, if not denial (Langer, 1994).

Langer (1994) reports incidences of individuals who show little distress following traumatic or catastrophic disabling losses. She brings up the question of whether they are simply minimally distressed by their situation, have not realized the full impact of these losses, or whether they are in a state of denial. Wortman and Silver 
(1992) report on their own research involving longitudinal studies assessing how people cope with loss. One study assessed the frequency of postive and negative affect of individuals physically disabled as a result of a spinal cord injury. The other study measured the emotional responses of parents who suddenly and unexpectedly lost a child to Sudden Infant Death Syndrome (SIDS). They concluded that low initial distress could not be dismissed as simply denial. Their data did not support the widely held assumption that people who failed to show the "normal" pattern of loss were destined for subsequent health difficulties or symptoms. The studies also provided evidence that an initial period of depression following loss is not universal.

Trieschmann (1988) reviews numerous studies on the evidence of depression and denial in her book on spinal cord injuries (SCI). She differentiates grief from depression, depression classically characterized by loss of appetite, insomnia, and psychomotor retardation. Trieschmann argues the term grief more appropriately describes the sadness and loss experienced when a person becomes suddenly disabled. She concludes that depressive reactions are not a universal reaction to the onset of SCI and the absence of depression is not an indicator of poor 
adjustment to disability. Each study she reviewed noted individual differences in emotional response to SCI.

Biological factors may account for some of the depression experienced in disability (Friedland \& McColl, 1992). There may be some factors that are genetic and predispose an individual to affective disorders, the onset of disability acting as a precipitant to depression. Structural and neurochemical changes that are a part of the disease process may manifest themselves in structural changes in the nervous system, causing the individual to become more susceptible to depression. Another relationship may be between the medications prescribed for the illness or disability. Many drugs used for the treatment of physical disorders are known to have depressive side-effects. Friedland and McColl (1992) suggest that it is likely that there is an extremely complex relationship between biological and psychosocial factors to explain depression in the disabled population. Stewart (1994) combines the psychoanalytic and the cognitive viewpoints to form a working definition of denial in the rehabilitation setting. Denial is viewed as an ongoing process against anxiety which prevents disabling conditions and their consequences from being brought into awareness. Stewart conceptualizes denial as being on a continuum of defenses, its use influenced by the 
individual's style of relating, world view, personality, and by the amount of anxiety resulting from the disability. The scope of denial and its significance are specific to each individual.

According to Langer (1994), denial may be a positive initial response to trauma, serving to protect the individual from becoming psychologically overwhelmed. Langer refers to this response as the psychic equivalent of shock. Denial of disability has a negative value if it is maintained over the long term, often being labeled as pathological (Stewart, 1994). Trieschmann (1988) reports that it appears denial is not evident in most cases in which an individual becomes suddenly disabled. She believes "hope of recovery statements" are sometimes confused with denial (p. 85), that statements that may appear to indicate denial may actually be positive indicators of hope. Langer (1994) stresses the importance of hope and maintaining hope to avert despair and to preserve "a sense of continuity and meaningfulness in life itself" (p.192).

Turner and Noh (1988) conducted a longitudinal study to try and determine the association between disability and the risk for depression. They found physical disability posed a dramatic elevated risk for depression regardless of gender or age. Their data revealed that chronic strain 
becomes increasingly relevant with age, while life events became less so. The number of life events tend to vary by life stage, evidence showing that older people experience fewer events. Social support and mastery showed strong and consistent relationships to depression in all age groups, suggesting that they might be promising intervention targets. Other studies have revealed there are aspects of social support thought to modify the effects of traumatic loss and ease recovery from bereavement (Osterweis et al., 1984). Examples of these social supports are enhancing self-esteem, problem-solving, networking, and providing relationship resources for meeting life cycle transitions.

\section{The Debate Concerning Stage}

\section{Models}

The committee appointed by the Institute of Medicine to review bereavement cautioned against the word "stages" to describe the bereavement process (Osterweis et al., 1984). They believe the term could suggest the existence of concrete boundaries between what are actually "overlapping, fluid phases." The bereaved person might be treated inappropriately because of expectations of where the individual is or ought to be in the grieving process. Wortman and Silver $(1987,1989,1992)$ have reviewed studies on coping with stressful events. They claim that the data do not support, and sometimes contradict the stage 
approach. According to Wortman and Silver, stage models are often presented in ways that are difficult to disconfirm and are hard to test empirically. Stage models are sometimes used as measures of progress to evaluate how a person is doing (Wortman \& Silver, 1992). The person may be judged as reacting abnormally or inappropriately if he or she does not "work through" loss in the expected pattern (Wortman \& Silver, 1987).

Attempting to force a person through the stages of grief is insensitive to the needs of the grieving person and makes the personal experience into a mechanical process (Karl, 1987). Wortman and Silver (1987) contend there is little empirical evidence to support the concept of "working through." They report that those who display the most signs of working through the loss are those who ultimately have the difficulty resolving what has happened. Most stage models assume individuals will confront their loss and go through an intense period of distress or depression (Wortman \& Silver, 1987, 1989). Wortman \& Silver's (1987) examination of research failed to find evidence that depression is inevitable following loss. They have also challenged the concept of a universal reaction to loss claiming that stage models are unable to account for the diversity of outcomes occuring in response to loss. Stage models fail to offer the reasons why one 
person is relatively unaffected by a loss event while another may be devastated by a very similar experience.

Kübler-Ross has been a particularly popular target of attack from those who are critical of stage models of adaptation (Corr, 1993; Karl, 1987; Schulz \& Aderman, 1974). Klass (1981-82) feels that Kübler-Ross' work has been accepted more on an intuitive basis - because it feels right to the reader. Many people refer to her stages of grief as if they were a recipe for health (Karl, 1987). Others say it is inadequate, superficial, and misleading with no conformation of its validity or reliability (Corr, 1993). The only conclusion researchers have in common with Kübler-Ross is that most terminal patients experience depression shortly before death (Schulz \& Aderman, 1974). Schulz and Aderman (1974) report kübler-Ross' work is highly ambiguous, is the product of highly subjective subject matter, and lacks specific assessment procedures for determining what stages a patient has passed through. There are questions as to whether grief is ultimately achieved and whether it is a universal process (Wortman \& Silver, 1989).

Corr (1993) finds kübler-Ross' stage-based model as lacking in dimension, its dynamics mainly involving defenses responses to threat and conflict, and as failing to explain how a person passes from one stage to another. 
He offers three reasons not to focus on the stage-based model drawn from the work of kubler-Ross. First, there is no reason to assume there are only five ways in which to cope with death. Second, there is no reason to believe that these five basic forms should be or somehow interlinked as stages in a larger process. Rather than stages, they might be more accurately classified as discriminable coping strategies. Third, there is no evidence that the five stages identified by Kübler-Ross are somehow obligatory ways in which a person must or should cope with dying. Corr (1993) feels a model for coping with dying should foster empowerment and emphasize participation of shared aspects of coping with dying.

In all fairness to Kübler-Ross, some of the criticism against her is unwarranted due to the misuse of her model (Corr, 1993). According to Corr (1993), misinterpretation of Kübler-Ross' stage model has led to stereotyping dying people in ways that are inconsistent with the intentions of the author. Corr places the blame on reliance on what he terms "an inadequate theoretical model." Kübler-Ross never intended that the stages be viewed in such a linear fashion (Ahmed, 1992). She has suggested that many people do not flow from stage one to five in a chronological order (Kübler-Ross, 1974). Kübler-Ross believes the goal should be to find out where the person is, and then determine how 
he or she can best be helped rather than guided through the five stages and reach the stage of acceptance. Even though Kübler-Ross was reluctant to use the term stages, she failed to move on to other language or to improve her theoretical formulation (Corr, 1993).

\section{Value Change Theory of Acceptance to Loss}

Dembo, Leviton, and Wright (1956) viewed acceptance of loss as a process involving changes in the value structure of the person rather than becoming reconciled to an unfortunate situation. Disability can take on a meaning that can lead to underestimating abilities and potentially to a devaluation of the entire person. Acceptance of disability is seen as an adjustment of one's personal value system to the point where the value of existing abilities is not negated by actual or perceived losses from disability (Keany \& Glueckauf, 1993). Wright (1983) defines loss as "the absence of something valuable, experienced as a personal misfortune" (p. 163). Acceptance means viewing the loss as nondevaluating. Wright looks at reactions to disability in a social-personal perspective that integrates responses to social-environmental pressures and to personal processes (Shontz, 1980).

Wright built on the foundation laid by Dembo, Leviton, and wright (1956) identifying four major changes in the 
value system that prevent or limit devaluation (Wright, 1983). They are: (a) enlargement of the scope of values, (b) subordination of physique relative to other values, (c) containment of disability effects, and (d) transformation of comparative-status values to asset values. Wright (1983) contends that these value changes lead to an acceptance of ability that is nondevaluating and "free people to act in ways befitting their own characteristics rather than those of a normal idolized standard" (p. 183). Enlargement of the scope of values. Wright (1983) sees the period of mourning as a time characterized by preoccupation with the loss and by coming to terms with the 1oss. She differentiates this from the requirement of mourning which occurs when the observer exaggerates suffering on the part of the person considered unfortunate in order to protect highly held values. The able-bodied person might imagine how he or she would feel if disabled and might impose personal expectations of suffering and distress upon people with disabilities. The individual might have the motive of preserving his or her own status of superiority by overstating the suffering of a person considered unfortunate. Another type is the ought requirement of mourning which arises from a need to preserve codes of appropriate behavior, maintaining how one ought to feel and act. 
The period of mourning as a healing time in which "the wound is first anesthetized, and then gradually closed, leaving the least scarring" (Wright, 1983, p. 170) . Wright believes that people will experience mourning in different ways. She conceptualizes mourning as being on a continuum. Some individuals experience little distress, while others experience all-inclusive suffering. Mourning is a transition period that may help prepare the person to meet the challenges that are before him or her. Wright sees mourning as a state that is experienced intermittently rather than one that is passed through and left behind. The enlargement of the scope of values is the first value change to emerge when preoccupation with loss is intense (Keany \& Glueckauf, 1993). Simply stated, the individual learns that disability is not the only thing that matters. Values thought to be lost are rediscovered. The necessity of managing activities of daily living may help redirect the focus of the individual from the past to the here and now (Wright, 1983). The person may become satiated, tired of mourning, ready for something new. He or she may begin to recognize personal assets in seeing others with disabilities. All these experiences help the person emerge from mourning with the new realization that all meaning to life is not lost by the disability. 
Subordination of physique relative to other values. Although the person may have gained some satisfaction in enlargement of the scope of values, he or she may still remain troubled (wright, 1983). Wright attributes this to the excessive value placed on physical normalcy in our society and because other values are given insufficient weight. As mourning moderates, the scope of values widens to include values still available to the person from which he or she can gain satisfaction. Devaluation will be lessened when personality traits become more important or physical normalcy becomes less important - when the individual realizes other values are more important than physique. There is a shift from physique values to personality values over which the person has more control. Containment of disability effects. Dembo et al. (1956) used the term spread to describe how a single powerful characteristic can contribute to deductions about a person (Wright, 1983). Central to the values change theory is the contention that disability is only one of an assortment of factors determining the nature and direction of life. Not all areas of life are influenced or determined by the disability and not all the effects are negative. Those areas that are affected can be held within the more limited areas that actually involve the disability. Although disability may be a part of the 
person's identity, it does not identify the essential core of the person. Wright (1983) sums it up, stating that in certain situations, disability involves certain limitations, the limitations due to barriers imposed not only by personal incapacities but also by society. Transformation of comparative-status values to asset values. Status or comparative values involve judging the attributes of a person against a standard (Wright, 1983). The same attributes may be evaluated in other terms based on such terms as usefulness or intrinsic value. This is referred to as asset evaluation. This dual approach to assessment could result in something being viewed as below standard by status judgments at one extreme or being seen as valuable when evaluated by asset value standards at the opposite extreme. To illustrate this point, Wright (1983) uses the example of the ability to use crutches as being an asset to mobility rather than being classified as a symbol of inferiority because the person falls below normal standards. A healthy and whole body can be an asset, but at the same time it is not devaluating if a person does not meet higher comparative standards (Keany \& Glueckauf, 1993; Wright, 1983)

Dembo et al. (1956) used the concept of as if behavior to describe the "temporary forgetting" experienced by many people facing disability. Should the injured person and 
others forget the disability, there would be no injury and no difficulties associated with it. Dembo and her research team saw value in acting as if because it provided a temporary escape from the issues associated with the situation. While this mild denial of disability might help in keeping others from intruding into the person's privacy, it could also create problems in close interpersonal relations.

Even though the disability may not be able to be hidden, the person may act as though it makes no difference (Wright, 1983). The disability may be viewed as a punishment or as a threat because it jeopardizes the indivual's concept of the normal, nondisabled ideal. Wright maintained that maladjustive reactions are a necessary and an important step during the adaptation process. It is an effort to raise self-esteem by protecting oneself from the difficult psychological states of shame, self-pity, and inferiority. After time, the person becomes aware of the strain that nonacceptance of the disability produces. He or she realizes that one can live on satisfactory terms with the disability, finding comfort and reward in being oneself.

Although they feel the work of Dembo et al. (1956) and Wright (1983) to be important, Keany and Glueckauf (1993) maintain the major premises of their acceptance of loss are 
largely untested. They contend methodological inadequacies have limited prior research. Some of the inadequacies are the use of analog procedures rather than field tests, underreliance on samples of disabled persons, and limitations with using the Acceptance of Disability scale (Linkowski, 1971) to validate studies. Keany and Glueckauf (1993) could not find any studies that empirically examine all four values changes outlined in the theory of acceptance of disability. Value change is critical to the theory of the process of acceptance proposed by Dembo et al. (1956) and Wright (1983). Keany and Glueckauf (1993) report the four value changes are not well defined or operationalized leading to substantial measurement problems. They conclude that in order for the field to advance, research must look beyond "global evaluations of acceptance of disability during a single point in time to intensive longitudinal analyses of the social psychological processes linking onset of disability, value change, and acceptance of loss" (Keany \& Glueckauf, 1993, p. 209).

Keany and Glueckauf (1993) propose using Rokeach's Value Survey (1973) as an alternate way of clarifying the make up and meaning of value change after the onset of disability. In Rokeach's model, values are categorized as either terminal or instrumental. Terminal values are idealized end-states of existence, while instrumental 
values are the way a person acts to achieve his or her terminal values. The value system is conceptualized in a hierarchical fashion, the relative importance of a value determining its position to other values. Acceptance of disability consists of the reorganizing the order of the two types of values to accommodate changes brought on by the disability (Keany \& Glueckauf, 1993).

\section{Chronic Sorrow}

Olshansky (1962) introduced the term chronic sorrow to conceptualize response to ongoing loss. He used the term to describe the intense, recurring sadness he observed in the parents of mentally retarded children. The degree of sorrow may vary from time to time for the same person and from situation to situation. It may be influenced by such factors as personality, ethnicity group, religion, and social class. Olshansky (1962) repeatedly stressed that chronic sorrow is a natural, rather than a neurotic response to a tragic event. When a loss goes unresolved, such as in chronic illness, the sorrow the individual feels over time becomes chronic (Hainsworth et al., 1994). At times the sorrow may be dormant, but may be activated by reminders of unfulfilled life goals. Lindgren, Burke, Hainsworth, and Eakes (1992) make a distinction between the concepts of chronic sorrow and prolonged grief. While 
prolonged grief is a long-term reaction to one loss, chronic sorrow is the reaction to multiple losses over time.

Davis (1987) argues that stage theories of loss distort the grief experience of people who live with disabilities and limit the understanding of the psychology of mourning. She believes chronic sorrow better describes the grief experience of those with disabilities. Disability results in mourning that is cyclic or recurrent and facilitates or requires coming to terms with "a changed, suffering, self" (Davis, 1987, p. 352). Supporters of the chronic sorrow model agree that there are some identifiable reactions to loss, such as denial, disbelief, isolation, bargaining, sadness and anger. They debate the viewpoint of adjustment to sorrow as being a time bound process ending in resolution (Burke, Hainsworth, Eakes, \& Lindgren 1992; Davis, 1987). Chronic sorrow differs in that there is no predictable end (Lindgren et al., 1992). The chronic nature of the disability serves as a constant reminder of loss, with both external and internal forces triggering the feelings of loss, disappointment, and fear. Burke et al. (1992) see chronic sorrow as a normal reaction to significant loss, the person typically highly focused and functional in the presence of 
ongoing loss in opposition to pathological grief which can result in dysfunctional behavior.

In an attempt to validate the concept of chronic sorrow, Wikler, Wasow, and Hatfield (1981, cited in Teel, 1991 ) surveyed parents of mentally disabled children and social workers working with the families. Both groups described the periodic, recurrent sadness reported earlier by Olshansky (1962). They also confirmed Olshansky's findings that the sorrow did not increase in intensity and was especially evident during critical developmental stages of the child. A subsequent study by vines (1986, cited in Lindgren et al., 1992) supported the cyclical nature of the feelings of fear, sadness, guilt, anger, and helplessness experienced by the parents of mentally retarded and physically disabled teenagers. A resurgence of these feelings was reported by the parents as their children failed to complete developmental tasks. Hainsworth et al. (1994) conducted a qualitative study to examine the presence of chronic sorrow in individuals with chronic or life threatening conditions. The participants told of feelings of sadness, guilt, anger, frustration, and fear which occurred periodically throughout their daily lives since the onset of their disability. Hainsworth et al. (1984) concluded that chronic sorrow seems to be a normal state in chronic or life-threatening conditions and 
may occur in both the person with the condition and in family members.

Zisook and DeVaul (1985) believe their studies suggest that grief is an overly simplistic concept. Most people never totally resolve their grief, some aspects of the loss process continuing for years in otherwise normal patients. Parkes (1985) states that it is not possible to adopt a rigid time frame for bereavement, each relationship is different and each grief is different. According to Parkes, there is no clear end to grief, the past not completely forgotten. The individual gradually discovers which assumptions are still relevant and enhance his or her new life and which ones must be abandoned. Wortman and Silver (1987) argue there is sufficient evidence to contradict the common assumption that the individual suffering a loss will achieve a state of recovery and return to "normal" functioning with the passage of time. In reality, many people continue to show distress for much longer times than expected and some may continually reexperience the loss for the remainder of their lives. In their study on coping with SIDS, they found minimal evidence that resolution increases over time. Research conducted by Shadish, Hickman, and Arrick (1981) suggests that all disability related loss is not erased in the adjustment process (Marshak \& Seligman, 1993). Individuals 
with spinal cord injuries, some who had been injured for 18 years, continued to think about and "really miss" basic activities they used to enjoy. Marshak and Seligman (1993) report that some of their clients still experienced some sorrow related to their loss even though they had found deep satisfaction in their lives since injury. There are two events that must occur before the onset of chronic sorrow (Lindgren et al., 1992). First, there must be a trajectory of chronic illness or disability. According to Lindgren et al. (1992), the trajectory includes what led up to the onset of the illness or disability, what is currently happening, and what may or may not happen in the future. The person experiencing chronic sorrow may be either the one who becomes ill or disabled or a caregiver to an individual who is ill or disabled. Second, there must be an identifiable beginning to the trajectory such as the birth of an ill child, a diagnosis of a chronic illness, or onset of a disability. According to Teel (1991), the defining attributes of chronic sorrow are episodic pain and sadness. This pain and sadness a) vary in intensity at different times for the individual, b) are permanent and recurring during the lifetime of the individual, and c) are interwoven with periods of neutrality, satisfaction, and happiness. 
Kowalsky (1978) uses the term "trigger experiences" to refer to events which set off the grief process all over again. According to Kowalsky, if grief has already been worked through, it will be relatively short-lived the second time around. In cases where mourning has not previously occurred, more trigger experiences will take place. Kowalsky (1978) presents individuals with disabilities as having to come face-to-face with their altered selves and limitations at the time of these events. They begin to recognize how different this new image is from what they had hoped for in their future both before the onset of disability and even after the condition occurs.

\section{Cognitive Adaptation Theory}

Most people facing personal tragedy, such as illness or death of a loved one, do adjust quite successfully, returning to or exceeding their previous level of psychological functioning (Taylor, 1983). They typically rely on social networks and individual resources rather then seek professional help. It is these self-curing abilities that led Taylor (1983) to propose a theory of cognitive adaptation to threatening events. Taylor contends the process of readjustment centers around three major themes of a) a search for meaning in the experience, 
b) an effort to gain mastery over the event, and c) an effort to regain self-esteem. While Taylor's research focused on breast cancer patients, she believes the theory is applicable to other threatening events.

The search for meaning involves finding out why a crisis has occurred and understanding what impact it has had on the person's life (Taylor, 1983). Meaning may be addressed through causal attributions. Attribution theory claims that people make attributions after a threatening or dramatic event in an effort to understand, predict, and control their environment (Wong \& Weiner, 1981, cited in Taylor, 1983). Understanding why something happened may lead to understanding the significance of the event and what it means about the person's life. Taylor (1983) found in her research that $95 \%$ of the 78 women interviewed offered some explanation for why their cancer occurred and that no one attribution resulted in better adjustment. The search for meaning also involves a restructuring of one's life along more satisfying lines. Individuals often reorder their priories giving higher precedence to relationships, personal projects, or just enjoying life (Taylor, 1983).

The theme of mastery centers around trying to gain a feeling of control over the threatening event and over one's life (Taylor, 1983). In the cancer patients, the 
belief in direct control of the disease was quite strong. The patients used direct efforts such as meditation, imaging, self-hypnosis, positive thinking, and dietary changes to enhance the feeling of personal control. A sense of mastery was also gained by gathering information about cancer so the individual could become a part of the treatment. Taylor believes that self-generated feelings of control over a chronic condition enhance coping.

The third theme is the process of self-enhancement. According to Taylor (1983), research has documented that a drop in self-esteem is typical following a threatening event even if it is believed to have been caused by forces beyond the control of the individual. Taylor (1983) claims self-enhancement occurs when the person finds personal benefit from the experience, from comparing oneself with others who are less fortunate, and by concentrating on aspects of one's personal situation that make one appear to be well off. She found most of the patients used downward comparisons, comparing themselves with others who were as fortunate or less fortunate than themselves. If a comparison person was not available from personal experience, the patient created a norm where others were worse off than she was. Selecting a role model who is physically disadvantaged but successfully coping helps the 
person to feel better about oneself and provides a model on which to pattern efforts at readjustment (Taylor, 1983).

Taylor (1983) maintains that forming and maintaining a set of illusions is fundamental to resolving the three themes of meaning, mastery, and self-enhancement. She defines illusions as beliefs that have no factual basis or as looking at the known facts in a particular way. While illusions are often associated with defensiveness in the psychological community, Taylor claims they can have a dynamic force and may be essential for adequate coping. Denial may shield the individual against crisis both in the initial stage of threat and periodically as people are faced with information that is hard to accept. silver and Wortman (1980) found that the unrealistic beliefs of quadriplegics and paraplegics concerning their personal effort and their likelihood of recovery often led to better emotional functioning and better coping (cited in Taylor, 1983). Taylor (1983) concludes that the most effective individual at the time of crisis is the one who permits the development of illusions, nurtures them, and is eventually restored by them.

The Stress and Coping Model

The "normal" way to cope with irrevocable loss is commonly viewed as a process beginning with intense 
distress that gradually dissipates (Wortman \& Silver, 1987). Assumptions about loss are based partially on theory, partially on clinical lore, and partially on how culture dictates people should react to loss. After reviewing the literature, Wortman and Silver $(1987,1992)$ have challenged some commonly held assumptions about loss. They concluded the available research has failed to prove that a) depression is inevitable following loss, b) people experience both distress and positive emotions subsequent to loss, c) a period of depression is essential for successful resolution of grief, d) those who fail to show early distress will experience later difficulties, e) working through is important to loss and, f) continued attachments to the lost loved one are adaptive or maladaptive in the long run. They did find evidence to support that there are individuals who continue to show distress for a longer time than would be commonly assumed, some reexperiencing the loss for the rest of their life and that people are not always able to resolve their loss. Wortman and Silver (1992) reached the conclusion that stage theories do not account successfully for the variability of outcomes in response to loss. They fail to explain why one individual may be devastated by a loss while another person may emerge relatively unaffected by a similar type of loss. 
According to Wortman \& Silver (1992), the stress and coping model is based on the work of two theorists, Cannon (1939) and Selye (1956). Cannon and Selye hypothesized that changes in life create disequilibrium, imposing a period of readjustment. This period of readjustment can increase the individual's vulnerability to stress and its consequences. This holds true for both negative and positive life events. Despite extensive research, there is only modest evidence supporting a relationship between life change and subsequent disorder. As a result of these data, current research has shifted to the identification of factors that can explain the variability that exits in response to stress. Dohrenwend et al. $(1978,1981$, cited in Wortman \& Silver, 1992), developed a general model of the life stress process.

The model has been highly influential during the past decade (Wortman \& Silver, 1992). In general, it is assumed that the individual's vulnerability or resistance factors will determine how a stressful life event is appraised as well as its influence on subsequent mental and physical health. According to this model, those with more coping resources will probably recover faster and more completely than people who have fewer resources. Wortman and silver (1992) see this as an explanation for the variability in 
response to loss, an area left unaccounted for in stage models.

Wortman and Silver (1992) report drawing heavily from the stress and coping model in their personal work. One study involved interviewing 125 individuals who became physically disabled following an accident (Silver, 1982, cited in Wortman \& Silver, 1992). Emotional response to the injury was measured based on the affective states of anger, depression, anxiety, and happiness. The study showed that even though people do experience distress following a loss, they also experience positive emotions. The disabled subjects reported less happiness than the normative sample, but the difference was not significant. The injured individuals experienced happiness at least as frequently as feelings of anxiety, depression, and anger.

A second study on how parents cope with the loss of an infant to SIDS was attempted to replicate the previous results (Wortman \& Silver, 1987, 1992). Data showed that at both 3 months and at 18 months after the loss, positive affect was more prevalent than negative affect. Wortman and Silver (1992) also found that many of the parents (26.28) did not demonstrate intense depression at either of the two assessment periods. They contend this data counters the popular belief of the inevitability of depression or emotional distress following loss. Wortman 
and Silver (1992) concluded that these two studies provide evidence that people not only experience distress following a loss but positive emotions as well. Stroebe (1992-93) questions the validity of the SIDS study. Wortman and Silver $(1987,1989)$ used the term "working through" to identify active attempts by the parents to make sense of and process the death of the child. Searching was found to be related to poor outcome. Stroebe (1992-93) believes that what Wortman and Silver termed "working through" is at least in part, "renumination" which could lead to alternate interpretations of the data.

Wortman and Silver (1992) are attempting to develop a model that accounts for their findings of the striking variability in response to loss and that a significant number of individuals do not appear to experience intense distress following a loss. Their basic premise is that following a loss, the impact on subsequent health and functioning is largely dependent on whether the loss can be incorporated into the person's world view. Wortman and Silver (1992) hypothesize that individuals who can integrate the loss into their view of the world would most likely experience much less distress than people whose world views are shattered. They also expect that people who have significant coping resources, and who have usually been rewarded for their efforts, may be especially 
susceptible to certain types of sudden, undesirable life events. A loss that challenges the viewpoint that efforts are generally rewarded might potentially be more devastating to such people than those who have considerably less coping skills (Wortman \& Silver, 1992). Wortman \& Silver (1992) are conducting research that tries to measure individual world views prior to undergoing a major loss. They believe this will help clarify the conditions under which various views are protective in the event of loss. Cheikin (1981) previously presented an outlook on loss which is similar to the world view model proposed by Wortman and Silver (1991). He contends that in all loss, the severity of the trauma directly relates to the degree to which the person's reality system is attacked. According to Cheikin, the reality system defines how one views self and the world and dictates how one relates to the world. It is formed as the individual proceeds through the activities of everyday life, beliefs evolving as he or she evaluates how well these experiences fill needs. Each loss and change in life presents a challenge to the reality system and provides a rehearsal for anticipating future losses. It is a natural tendency to hold on to established realities, resisting change at almost any cost. Cheikin asserts that the deeper or more central the belief is to the core of the person, the greater the trauma to the whole 
reality system and the greater the pain from the loss. Each attack on the reality system, each loss, creates a vacuum, leaving the person temporarily ineffective and disorganized. The void is filled as the individual sorts out the implications of the loss and reestablishes his or her reality system, turning a painful ending into a rich beginning.

\section{Developmental Theory}

Sigelman and Shaffer (1991) define development as the systematic changes in the individual that take place between conception and death. These changes include physical development, cognitive development, and psychosocial development. The following basic premises have evolved from the life span perspective on human development. Development is not limited to childhood, but occurs throughout the life span. It must be viewed in the historical and cultural context in which it occurs. Development can take many directions, each individual experiencing both systematic gains and losses throughout life. Development is influenced by a wide range of factors, both inside and outside the person, which interact with each other. Development is best understood if changes in one period of the life span are viewed in relation to changes in other periods of life. 
A number of theorists have addressed the concept of human development. Freud (1905/1953) believed the behavior of humans was biologically based and identified five psychosexual stages. Erikson (1959) offered a psychosocial approach in which the individual progress through eight stages of conflict resolution. Piaget's (1936/1950; $1947 / 1963$ ) theory is cognitively based, stressing four distinct stages that must be accomplished if one is to make sense of the world. There are several basic tenets common to all these approaches (Breger, 1974, cited in Whitt, 1984). They are a) the lifestyle evolves in stages rather than at a steady rate, b) the stages of developments follow a predetermined order, c) no stage can be successfully bypassed, d) each successive stage qualitatively alters the individual's capacities for interaction with and adaptation to the environment, and e) each stage is a more complex, differentiated, and integrated version of the developmental phenomenon preceding it.

In looking at a stage theory such as Erikson's (1959), healthy development involves mastering the tasks of each life stage before passing to the subsequent stage (Sutkin, 1984). Relative competence in a stage is a prerequisite to facing the challenges of the next stage. This requires meeting the minimum standards for the physical, emotional, and cognitive capabilities of each stage. An external 
threat, such as disability, can upset equilibrium and may result in a temporary fixation at a stage of development or in regression to an earlier one (Whitt, 1984). Some disabling conditions could diminish the smooth transition from stage to stage or might diminish the capacity to master tasks considered important to the development of a healthy ego (Sutkin, 1984). If later development depends on successful completion of the prior stage, the individual may be hampered in approaching the subsequent challenges of life. Looking at disability in relationship to the life span calls for the integration of developmental milestones and happenings into the meaning of and reactions to the disability (Burke et al., 1992). Age would appear to be an important variable in considering how people with disabilities might react to loss (Sutkin, 1984). The physical and cognitive development, the expectations, the accomplishments, the needs, and the responsibilities of the adult will differ from those of the child and the elderly. A more in-depth discussion of the variables influencing loss will be presented in the next chapter. Piaget $(1936 / 1950 ; 1947 / 1963)$ has identified four distinct stages of cognitive development, each successive stage building on the previous stage and representing a more complex way of thinking. The sensorimotor stage involves using sensory and motor schemes to explore and 
understand the world. In the preoperational stage, symbols are used to gain an increasingly complex way of viewing the world. The concrete operations stage is characterized by the use of logic and an ordered mental manipulation of the symbolic representations of events and objects. In the final stage of formal operations, the person can engage in reflective thought that includes abstract ideas, possibilities that do not exist, and long range Consequences (Lerner \& Gignac; 1992, Sigelman \& Shaffer, 1991).

Lerner and Gignac (1992) have looked at how these four stages might operate in the event of disability. They use reactions to cancer to illustrate their model. There are three levels of "knowing" and three concurrent sets of associated emotions related to the diagnosis of cancer. The reactions typically range from the simplest direct experience of the pain to the increasingly complex grieving reactions that appear as the meaning of the event becomes clear to the thoughtful examination of what the label of cancer implies. At the pre-symbolic level, tactile cues and pain sensations predominate. Experiences at this stage might involve coping responses such as denial. By simply not acknowledging the cancer, the victim finds relief from the aversive states of pain, suffering, and anxiety. 
At the symbolic level of concrete operations, the individual may relabel his or her fate to minimize suffering or find compensating benefits. Despite the severity and its implications, the disability may be seen as a benefit in the person's life. Aversive emotions associated with this period are often a combination of fear, anxiety, anger, and resentment. The metarepresentational level of formal operations includes a thoughtful examination of what the disability implies in the individual's life. Negative emotions associated with this phase are guilt, grief, dread, and demoralization. Positively, the individual may generate more comfortable causal explanations for his or her suffering, or find compensation by redefining what the future holds (Lerner \& Gignac, 1992). Lerner and Gignac see this pattern of avoidance, and/or redefinition of the crisis to deal with the pain, anguish, and suffering, as being likely to be repeated in reaction to the next crisis, illness, or loss. Continued reinforcement strengthens the reliance on these familiar coping responses, thus preventing the development of alternate ways of dealing with crises.

Brandtstädter and Renner (1992) describe a similar process of adjustment. They claim people attune their actions to developmental goals and may also adjust their system of goals to perceived developmental potentials and 
capabilities. Options that seem unattainable or blocked are often replaced with new goals. Brandtstädter and Renner (1992) view these strategies as a personal form of protection against permanent dissatisfaction, depression, and grief. There are two separate yet interrelated modes of coping with discrepancies between aspirations and actual achievements of life circumstances. The first is the assimilative tendency. In this process, an individual might actively try to alter the situation which is creating the discrepancy so that it might more closely mesh with personal goals and ambitions. Basically, this is a problem solving phase. Negative emotions arise from repeated failure to resolve the cause of the distress. They may range from initial reactions of anger, worry, or disappointment to feelings of helplessness and hopelessness to intense preoccupation, growing disorientation and depression in the critical stage. If attempts to alter the circumstances are unsuccessful or the price is too high, accomodative tendencies begin to dominate.

Brandtstädter and Renner (1992) see the accomodative process as more of a phase in which the problems are neutralized or dissolved rather than corrected. This mode involves a readjustment of the individual's developmental goals and life themes. It is a palliative reappraisal which involves a shift in the meaning given to a particular 
event. The individual may reject certain facts, doubt the correctness of information, or adopt alternate causal or theoretical beliefs. He or she may compare the situation to a hypothetically worse world. When compatible palliative reappraisals are unavailable, only a radical change of perspectives that involves a fundamental redefinition of world and self can create new and satisfying developmental perspectives.

The ability to flexibly adjust to life circumstances, to replace blocked goals, and to positively reinterpret adverse situations are seen as factors that contribute to successful coping with developmental losses. Brandtstädter and Renner (1992) propose that there is a gradual shift from assimilative to accommodative modes of coping in middle and later adulthood. They offer this as an explanation to how people are able to maintain a positive outlook even in the face of an increasing proportion of developmental losses over gains.

Lerner and Gignac (1992) maintain there is another developmental process, whereby, the elderly especially can achieve contentment with relabeling, distorting, or denying the reality of their circumstances. Rather than defining and redefining the event in the usual and comfortable way, it requires the person to step back and take ownership of the connection between his or her emotional reaction to the 
event and the perspective taken of the event. It involves adopting different perspectives of the same event, imaginably viewing these alternatives, recognizing that each perspective is a valid but limited view of the event, and finally reassessing and then choosing the perspective which is best for the person. While this process may not always result is less grief, suffering or anxiety, the person is no longer viewed as a helpless victim. Rather he or she is an active respondent to the events gaining control over personal experiences and freedom from the constraints of any one way of viewing present or past realities.

Weenolsen (1988) contends loss and separation are recurring themes throughout the life span of the individual. She sees loss as resulting in the destruction of one part of life and consequent re-creation of another. It is metaphorically related to death, loss representative of a smaller death of self and life. Every change, no matter how small, involves a degree of loss that must be overcome and result in a re-creation of life and self. As each loss diminishes the meaning of life, each transcendence of loss enriches the meaning of life.

Weenolsen (1988) draws from the work of Neugarten (1968) in her discussion of the effect of timing on the loss. On-time losses are losses that are expected at a 
certain time in the developmental stages. These are normative life events such as separation from the mother, starting school, graduating, looking for work, getting married, and having a baby. off-time losses are those typically occuring at a certain stage in life, but come at either an earlier or later stage. Weenolsen believes these type of Iosses tend to be more traumatic than on-time ones because they force the individual to question the predictability of the on-time losses. Time-irrelevant losses are losses that may or may not necessarily occur in a person's life and are not related to any specific developmental stage.

According to weenolsen (1988), there are five levels of loss: primary, secondary, holistic, conceptual, and metaphorical. The primary level refers directly to the specific loss incident. Secondary losses are associated losses which follow directly and with some immediacy after the initial primary 1oss. At the third level are the more remote and abstract losses to the life and self. Holistic losses might be represented by aborted plans for the futures, shattered hopes, and lost dreams. Conceptual losses, or losses to the self or self-concept, require a redefinition of self. At the fifth level are the metaphorical Iosses or the individual meaning attached to each loss relative to one's personal metaphorical system. 
Weenolsen (1988) believes that it is beneficial to look at loss from this tier perspective for two reasons. First, it helps people understand why loss can affect them so deeply. Second, loss can be dealt with at each level instead of seeking resolution on the primary level only. No one actually gets over or forgets a loss. Losses become a part of the individual, an essence of the self, as do the transcendence of these losses.

Weenolsen (1992) explains transcendence as being a two-step process. The first involves overcoming the loss in some manner. Next, aspects of life and self are structured in a different way due to the loss and overcoming. The processes of loss and transcendence make up the two phases of mourning and resolution. There are two stages in the mourning phase (grieving and searching) and two in the resolution phase (replacement and integration). In the grieving stage, there is a yearning for what has been lost and for replacement of what was lost. It is often begins with shock or numbness and may culminate in hopelessness. If replacement seems impossible, the individual may become stuck in this stage or progress may be delayed. Searching involves trying to find the replacement for what has been lost. The person will not move on to the next stage if no worthy replacement is discovered. Replacement means finding a substitute for 
the lost as equal in value as possible to but not exceeding the original loss. Even though the replacement is accepted, the loss may still be keenly felt and passage into the next stage may be slow. The stage of integration consists of incorporating the meaning of the loss into the self. This includes integrating the cause of the loss, feelings of guilt or helplessness connected with the loss, how one dealt with the loss, and ultimately how the self is changed or restructured because of the loss and its transcendence.

The stages are sequential but return to a previous stage is possible. There is often a pattern of back and forth movement. While the individual might progress from grieving to searching and then back to grieving, he or she would not skip to full integration. Weenolsen (1992) sees this reversion as reaction to a new loss, most likely in the perceptual, abstract, self-conceptual, or metaphorical loss levels. Much like the assimilation and accommodation processes proposed by Piaget (1952/ 1963), loss and transcendence from a single unified process. A dynamic equilibrium is established, one not possible without the other.

According to Weenolsen (1992), loss and transcendence is a life long process, occuring throughout the developmental stages. There are losses specific to birth, 
to infancy, to early and to middle childhood, to adolescence, to periods of adulthood, to senescence, and finally to dying. In any given developmental stage, losses may be on-time, off-time, or time-irrelevant. Weenolsen (1992) defines pathology as a) the inability of the person to transcend a loss, or b) transcendence of loss by another loss that does not lead to transcendence, or may even lead to greater loss. There may be inadequate transcendence at any stage of the loss and transcendence process. Consequently, the individual may become fixed at that stage resulting in pathology. Weenolsen gives the example of a man whose wife dies and becomes so preoccupied with her death that he is unable to move on to the next stage of searching. The man may isolate himself, lose interest in work and everyday activities, start drinking, or be unable to eat. If feelings of hopelessness or helplessness prevail, he may lose his desire for anything. In this case, the stage of searching becomes a futile effort. A person who becomes ill or disabled could become fixated at the replacement stage if he or she is unable to let go of the identity held before onset of disability. The individual may be reluctant to give up prior ideals and old concepts of significance and fails to consolidate the new identity. Weenolsen (1992) contends people who are 
stuck in the replacement stage display deepening and chronic anxiety.

The Existential Perspective of Loss

Many theorists of loss believe that the ultimate loss is life itself and that the ultimate threat is death (Shontz, 1980). Becker's (1973) views are more philosophical than psychological (Shontz, 1980; Stephenson \& Murphy, 1986). According to Becker (1973), everyone attempts to become self-sufficient by maintaining a superiority over death. Becker argues that the quandary of humankind today stems from its institutionalized denial of death. Modern humanity is ill-equipped to respond to questions about death. People escape the reality of death by creating a pseudo world which avoids death as much as possible. Any reminder of this reality must not be allowed into one's consciousness.

Becker's (1973) line of thinking may be readily applied to disability (Shontz, 1980; Stephenson \& Murphy, 1986). It offers an explanation why some people are poorly prepared to accept their disability and why many members of society avoid association with those who have disabilities. The chronically ill and disabled must both come to terms with their personal loss and must also face a society generally unsupportive of their condition and of their 
endeavor to bring meaning to their lives. Grief may be to some degree a response to a heightened fear of death, the disability symbolic of potential total loss (Stewart \& Shields, 1985). Confronted with the inevitability of death, the individual may experience the sense of aloneness in life and be overcome with anxiety (Friedland \& McColl, 1992). Disability may lead to feelings of emptiness and sadness, life robbed of its prior meaning he or she had so carefully created. Stephenson and Murphy (1986) maintain the effort to bring meaning to loss is a key element in the grief process and healthy adaptation.

The chronically ill and disabled are reminders to the members of society of their own mortality, a truth they would rather avoid (Stephenson \& Murphy, 1986). Society has responded by relegating the chronically ill and disabled to a life removed from the mainstream. This is yet another loss imposed on this population - the loss of social acceptance. Zola (1982, cited in Stephenson and Murphy, 1983) claims that individuals who are disabled or chronically ill are the victims of this process, often experiencing grief in response to their change of status.

It has been argued that separation is a deathequivalent in life (Lifton, 1979, cited in Stephenson \& Murphy, 1986). For people with disabilities, separation may be evident in both society's rejection of them and in 
the individual alienation from his or her former self. The limitations imposed by the disability and society may result in a less active and more passive role for the individual. Stephenson and Murphy (1983) identify two types of grief, existential and reactive. Existential grief is the sadness felt when the individual is unable to find meaning in loss. Reactive grief is the sadness experienced in response to loss of a loved one or valued object. They see both types operating in every loss, each at varying degrees. Grief may arise from a wide spectrum of losses including death, divorce, unemployment, a loss of a body part, and a loss of a part of the self, such as role or status.

According to existential theory, adaptation to disability or chronic illness involves moving through the grieving process over the loss of the former self. This is different from a normal bereavement process which is often characterized by active support from society. Rather than offering the support which is a crucial factor for successful grief resolution, society may instead abandon, set apart, or dehumanize the chronically ill or disabled. Guilt is a common emotion in the grief process (Stephenson \& Murphy, 1985). Taking personal blame for illness or disability can serve as an escape from the anxiety intrinsic in accepting the reality of death. By accepting 
responsibility for what has happened, blame has been established, and provides an escape from coming face-toface with the reality of death. Stephenson and Murphy (1985) contend that while the typical griever may be able to once again become a part of a society that holds tight to its avoidance tactics, this is not possible for the chronically ill and disabled. The reality of disease and death is ever-present and unavoidable. 


\section{CHAPTER IV}

\section{VARIABLES INFLUENCING RESPONSE TO LOSS}

In his paper on the process of mourning, Bowlby (1961) concluded that the phases he described apply to all ages, from the infant to the adult. He adds it would be wrong to assume there are no differences, age being a main variable in determining the course of mourning. Age, gender, personality, and culture have been shown in study after study to influence adjustment to loss. With the onset of illness or disability, even more factors must be considered in individual response and adaptation. Reaction to disablement depends in part on when and how it happens (Vash, 1981). Other factors influencing the impact of disability are the type of disability, its severity, the current life circumstances of the individual, inner resources, self-image, the degree of family and social support, the available technology, and government funding trends ( Falvo, 1991; Vash, 1981). These variables account for variation in type and intensity of reactions to loss and interact with one another creating yet another level of complexity (Vash, 1981). 
This chapter will focus on variables that may influence response to loss, first examining age, gender, and culture. The remainder of the chapter will examine other influences that are more specific to disability. Disability often results in a wide span of emotions ranging from fear, anger, and sorrow to relief and sometimes happiness (Vash, 1981). Anxiety about survival and periods of rage are experienced by almost everyone with onset of disability. Within this limited spectrum of emotions are infinite ways of expressing them. This chapter will be an effort to answer the question of human variation - what causes individual reaction and how.

Age

An important consideration when looking at the factor of age is how the needs and responsibilities of one agegroup differ from another and what personal resources the individual brings to the situation (Sutkin, 1984). A person who experiences disability in adulthood will have very different obstacles to face than the child with a congenital disability who has grown-up within the confines of medical treatment (Blacher, 1970). Theorists have stressed the importance of mastering tasks of each life stage before moving on to the next stage to ensure normal development (Sutkin, 1984). Each life stage has its own stresses and demands that are associated with age- 
appropriate skills and behaviors (Falvo, 1991). Illness or disability can create barriers to meeting these achievements. Viewed within Erikson's (1963) developmental framework, the differing demands of adapting to disability are a function of the life stage in which it first appears. A disabling condition may in varying degrees diminish the physical, emotional, or cognitive capacities of the individual and may disrupt the smooth transition through the current stage. This section will look at the life span, identifying developmental tasks of each stage and how disability or illness might effect successful progression through the stage.

\section{Infancy and Early Childhood}

According to Erikson (1963), the major developmental task of this stage is gaining a sense of basis trust versus mistrust (Sutkin, 1984). Not only do children begin to rely on their caretakers to be consistent and reliable but also learn to trust themselves and their individual capacities. The role of the mother (or caretaker) is crucial in this stage, she being available as a consistent and loving caregiver. Chronic disability in infancy resulting in hospitalization, and mother-child separation, may have an immediate effect on the infant's development. This disruption may result in a failure to thrive or problems in physical or intellectual development (Maddux, 
Roberts, Sledden, \& Wright, 1986). The young child may feel deserted by his or her parents (Blacher, 1970). In the period of early childhood, children develop a sense of autonomy along with an awareness and mastery of their environment (Falvo, 1991). They begin to learn social and communication skills essential to effective interactions with others. A child who is ill or disabled may have limitations that restrict physical activity, socialization, or exploration of the environment. This could be disruptive to the child's striving for confidence, mastery, and independence (Maddux et al., 1986). The child's limited capacity along with parental restrictions and protectiveness may prevent interactions that promote the important achievements of early childhood. An overly protective family member might restrict normal activities or an overly sympathetic parent may condone otherwise inappropriate behaviors. Constant restrictions followed by overindulgence may further complicate the child's development (Blacher, 1970; Falvo, 1991; Sutkin, 1984).

\section{The School-Aged Child}

When they enter school, children begin to expand their world beyond their immediate family, find their place in peer groups, and win recognition by their own efforts (Falvo, 1991; Whitt, 1984). They are exposed to a larger social environment, learning social relationships and 
interaction skills. It is a time when children develop a sense of initiative and industry. School-age children become aware of their special skills and expand their capacity for sustained effort that results in completion of tasks. According to Erikson (1963), failure to master this stage will result in pervasive feelings of inferiority and inadequacy, in a lack of confidence.

Self-image at this age is equated to the degree of skill the child believes himself or herself to have (Sutkin, 1984). For the first time in the child's life, the opinion of peers carry a greater weight than that of family in forming a self-appraisal. Children with physical or cognitive limitations may develop poor self-esteem if they believe their personal abilities fail to match the standard set by their peers. Peers may not accept the child who, because of illness or disability, lacks skills normally valued at this time (Falvo, 1991). Frequent absences from school because of their condition might reduce social interactions with peers. Parents, in trying to protect the child from emotional pain, may further isolate the child. Despite the good intentions of the parents, this could create the potential for reduced selfconfidence (Sutkin, 1984). It is essential the child experience interactions with peers and find acceptance from them to establish a sense of adequacy and competence. The child's adjustment is dependent upon the pre-disability 
level of cognitive and affective development, prior coping abilities, current parent-child relationships, the stability of the family, and on the meaning of the illness or disability to the child (Whitt, 1984).

Frears and Schneider (1981) point out factors that differentiate the child's reaction to loss from reactions of adults. First, children are rarely given the opportunity to learn about loss, adults believing they are sparing the feelings of the child by not sharing their own feelings. Another factor is that young children have not yet developed the cognitive capacity and psychological insight to understand and resolve loss. They will often form incorrect assumptions about what happened and may be unable to disconfirm them for years. Unlike adults, children usually do not have the means to resolve crisis, whether they be economic, emotional, or social. Adults make critical decisions for children which may leave them feeling helpless and hinder resolution of loss. Children who are hospitalized without being told the reason for it may believe they are being punished or sent away because of something bad they have done.

\section{Adolescence}

Wright (1983) offers three reasons why the period of adolescence should be given special consideration. First, it is a time when self-concept undergoes significant 
changes. Second, there is a heightened awareness of physique, physique playing an major part in the reformation of the self-concept. Third, adolescence is a transitional period, the individual being in an overlapping situation, "being a child on the one hand and an adult on the other" (p. 248). According to Wright, the adolescent with a disability has to deal with two kinds of overlapping situations. There are the situations directly attributable to disability and situations resulting from the transitional status as a child-adult. The battle of the wills, between the desire to be independent from parents and the need to be dependent can hinder the adolescent's adaptation to illness or disability and mastery of independence (Blumberg, Lewis, \& Susman, 1984).

Wright (1983) believes the circumstances of disability may at times prolong the period of economic dependence, postpone setting up an independent household, or prevent sufficient emotional separation from parents. In such cases, disability tends to draw out the adolescent period. Factors influencing the achievement of independence from parents are the nature and severity of the illness, the quality of the parent-child relationship, and the degree of acceptance of the disability by family members (Davis, Anderson, Linkowski, Berger, \& Feinstein, 1985).

According to Erikson (1963), adolescence is a time of search for identity. A successful outcome of this stage is 
the capacity for devotion and fidelity (Sutkin, 1984). The adolescent who has mastered the tasks of this stage will develop positive self-esteem, a comfortable body image, a realistic, goal-oriented view of the future, emotional independence, a sexual identity, and the ability to form positive relationships (Blumberg et al., 1984; Davis et al., 1985). Teenagers with disabilities must deal with the stress related to their condition as well as the stresses that normal adolescents face. Illness or disability at this time may disrupt relationships with peers. Limitations imposed by the condition and reactions by overly protective or restrictive parents may become barriers to the development of independence and individual identity. Rebellion against authority or the need of acceptance by peers, typical of most adolescents, can interfere with medical treatment associated with the illness or the disability (Davis, et al., 1985; Falvo, 1991).

There is a close link between acceptance of disability and mastery of many of the developmental tasks (Davis et al., 1985). Research has found a relationship between acceptance of disability and self-esteem and between the adolescents' perception of their body images and their ability to act autonomously. Adolescence is a period of strong preoccupation with body and appearance marked by avoidance of being "different" at all costs (Davis et al., 
1985; Falvo, 1991). Changes in appearance due to disability or illness could alter the adolescent's perception of body image and self-concept, possibly preventing the expression of sexual feelings. By learning to appreciate personal assets and avoiding comparison to others, the adolescent will more likely adopt values associated with the acceptance of disability (Davis et al., 1985). With self-acceptance will come increased self-worth and a strong sense of personal competence.

\section{Young Adulthood}

Following Erikson's (1963) life-span approach, the primary goal of young adulthood is forming intimate interpersonal relations (Glueckauf \& Quittner, 1984). Achievements include establishing oneself as a productive member of society, choosing a career, and accepting social responsibility (Falvo, 1991). Erikson believed individuals who failed to derive adequate measures of trust, autonomy, initiative, industry, and identity may have problems or be unable to form a genuine intimate commitment (Sutkin, 1984). Chronological age is not so much a factor for membership in young adulthood as psychological maturity (Sutkin, 1984).

It is also possible that the newly-disabled individual may fall back to already completed stages to allow for slow and safe adjustment to changes brought about by onset of 
disability (Sutkin, 1984). According to Sutkin (1984), there may be changes in trust levels, in one's sense of autonomy, in mental or physical capacities, or in sexual identity. For young people who have not yet established a meaningful relationship or independence, disability or illness may mean returning to their parents' home. Overconcern and sympathy may result in a situation that prevents the disabled young adult from undergoing experiences appropriate to his or her age group. Physical limitations may limit the ability to form intimate relationships and test already existing partnerships (Falvo, 1991). The ability to have children may be difficult, if not impossible.

There may be considerable variation in reaction to the disability or illness among family, friends and associates (Glueckauf \& Quittner, 1984). Such mixed reactions may leave the disabled individual questioning the sincerity and predictability of the response of family members and friends. If the person believes their responses are altered due to the disability or illness, he or she may choose to isolate himself or herself from social contact. Vocational choices may no longer be defined by interests or abilities but rather by limitations imposed by the illness or disability (Falvo, 1991). There may be a restricted range of job opportunities, transportation and architectural barriers, financial disincentives, and 
inadequate vocational rehabilitation services (Glueckauf \& Quittner, 1984).

\section{Middle Age}

In Erikson's (1963) view, the goal of the middle years is generativity. It refers to the capacity to care for others, willingly nurturing and guiding a new generation (Rustad, 1984; Sutkin, 1984). People in this stage are usually established in a career, have a committed relationship, and are assisting their own teenage children to become responsible adults (Falvo, 1991; Rustad, 1984). They may have to assume a new role regarding their aging parents, assuming more responsibility for them as they become fragile and dependent. It is a time that may require accepting and adjusting to the physiological changes that come with middle life. Individuals might revise goals as they begin to recognize the reality of their own mortality - that their time in this world is limited. Erikson (1963) believed it is essential that the individual have faith both in self and in the human race to successfully master this life stage (Sutkin, 1984).

Erikson describes those individuals who fail to achieve the tasks of middle age as being stagnant, being left in a state of personal impoverishment.

Neugarten (1977, cited in Rustad, 1984) has suggested the expected changes in mid-life, even though they may be 
negative, may not be sufficient to result in a crisis. Rather it is the "off-time" events, such as severe illness or disability, which are more likely to cause difficulty in adaptation. Disability or illness at this time could interfere with further occupational development, with the loss or reduction of the capacity to pursue vocational goals (Falvo, 1991; Sutkin, 1884). For some individuals it could mean early retirement. Family roles may be altered, the adult having to switch from being the nurturer to being nurtured or having to give up the role of provider for the family. Disability or illness might also have an impact on the individual's identity, self-concept, and self-esteem. Changes in sexual functioning may not only interrupt plans to have children, but may also affect both partners in an intimate relationship.

Cytrynbaum and Patrick (1979, cited in Power, Hershenson, \& Schlossberg, 1985) describe three developmental phases that take place as individuals attempt to adjust to change in middle age. Changes may be a result of biological decline, disability, a life-threatening illness, the death of loved ones, or social transitions such as early retirement or status loss. The first phase of reassessment begins once the individual realizes the irreversible nature of the change. At this time, there is a reevaluation of current identity and life structures. In the second phase of reintegration, the individual gains an 
increasing awareness of other aspects of personality. Defense structures are modified allowing for new, differentiated patterns of behavior and experiencing. In the final phase, behavioral and role change, there are recommitments, modifications, or significant changes in behavior or in relationships to family and/or work situations. According to Cytrynbaum and Patrick (1979), the individual who successfully negotiates the issues of each phase will emerge from the process with more individuality and greater self-confidence and selfknowledge than before.

\section{older Adulthood}

The successful outcome of the period of old age is ego integrity (Erikson, 1963). Erikson describes this period as an adaptation to the triumphs and disappointments of life (Sutkin, 1984). Ideally, older adults have accepted their own life and their imminent death (Falvo, 1991). Issues facing older adults differ dramatically from tasks of previous developmental stages (Sutkin, 1984). In most cases, vocational concerns are no longer a major concern. Dependent children are not usually an issue. The older adult begins to lose friends, partners, and familiar lifelong routines (Kivnick, 1985). There could be the threat of the loss of independence and dignity (Broden, 1970). Often, older individuals experience diminished 
physical strength and stamina as well as loss of varying degrees of sight and hearing (Falvo, 1991).

As the person loses the capacities of earlier years and suffers health problems that may lead to disability and handicap, despair may set in (Kivnick, 1985). A healthy psychosocial resolution requires that the individual draw from a lifetime of strengths to bring these feelings into a new, livable equilibrium. Strengths such as resilience and vitality help prevent impairments from growing into disabilities and disabilities from becoming handicaps. Broden (1970) views the reactions to the physical, social, and mental losses of old age as being on a continuum. At one end are the individuals who accept and compensate for their losses. At the other extreme are those who respond with clinical syndromes such as depression and hypochondriasis, covering the painful experience of loss. Broden (1970) believes the person's reactions to loss are primarily determined by experiences in early life, present personality characteristics, and the extent of the development of the capacity to deal with losses. A person who has high self-esteem, is self-confident, and actively faces life's challenges is less likely to develop feelings of helplessness and anxiety when confronted with the losses associated with old age.

In looking at the growth potential of the bereavement process of aging, Cassem (1975), suggests it is essential 
that the person give up some of his or her cherished delusions of power and greatness. At any age, almost everyone sets limits to the degree of physical and mental deterioration that is personally acceptable. Cassem (1975) describes this as the period when death seems preferable to life. As people grow older, they tend to expand the limits of acceptable deterioration. The person who is able to make such changes in tolerance to loss is much more likely to adjust to the losses experienced in old age.

This section has covered some of the gains and losses appropriate to stages in the life cycle. It has also focused on how disability might affect individuals in each life stage. The time of onset of disability was shown to potentially be a significant factor in determining how an individual might respond to the traumatic event. Wright (1983) has stressed the importance of taking age of onset of disability into consideration to help understand the life circumstances, tasks, and problems with which the individual must cope. She adds a word of caution, that age of onset, in itself, does not seem to be a decisive factor in determining the psychological outcome of the adjustment process.

Gender

When discussing gender it is often difficult to separate it from the factor of culture, the two often 
interrelated. This is especially true in our society in which men are under more pressure than women to "bear up" under tragic circumstances, keeping their feelings under control (Davis, 1987; Parkes, 1985). Some studies have revealed men have longer lasting problems after bereavement than women which may be due to the "macho" self-image that discourages expressions of grief (Parkes, 1985). The Tübingen Longitudinal Study (W. Stroebe \& M. Stroebe, 1993, cited in Stroebe, 1992-93) found individual differences between men and women. Men who held true to societal expectations, dealing with loss with "a stiff upper lip," have trouble in adapting. Those widowers who ignored role expectations and confronted their loss were able to work through their grief. Nolen-Hoeksema (1987, cited in Stroebe, 1992-93) offers an explanation she believes accounts for gender differences in depression. Women tend to display a more ruminative response style when depressed, which she contends boosts and prolongs their symptoms. On the other hand, men utilize more active response styles and engage in distractive behaviors when depressed. According to Nolen-Hoeksema (1987), this style of coping serves to shorten and stifle their depressive symptoms.

Gilligan (1982) questions the relevancy of traditional developmental theory when applied to women. She suggests that there is a bias against women in the life-cycle stage theories such as those presented by Freud, Piaget, and 
Erikson. Freud (1905/1953) noted the difference in women's development considering the variations to be a developmental failure in women. Piaget (1932/1965, cited in Gilligan, 1982) equates male development with childhood development. Erikson (1968, cited in Gilligan) noted that the sequence of development differs in females. For men, identity precedes intimacy and generativity in his conception of the cycle of human separation and attachment. For women, these tasks appear to be intertwined, intimacy going along with identity. Despite acknowledging this discrepancy, Erikson did not change his prediction of lifecycle stages, the experiences of men continuing to dominate his model. Gilligan (1982) believes the absence of women's experience in the formation of the theory of adult development distorts the conception of its stages and its sequence. Her goal is to increase developmental understanding by including the perspectives of both males and females.

Being male or female at onset of disability does not imply one or the other will have better or worse reactions, but rather different ones (Vash, 1981). One crucial element that may differentiate between the sexes is the greater acceptance by society of a passive, dependent lifestyle for women. Women, regardless of their prior degree of passivity or dependence, will be punished less by society for assuming these traits after onset of illness or 
disability. Despite this "advantage" over men, women with disabilities are more likely to be socially isolated, lacking in education, unemployed or underemployed, and have a limited income (Hanna \& Rogovsky, 1992). Women today are in a disadvantaged societal position due the oppressive messages sent to them by traditional socialization patterns (Alston \& MCCowan, 1994). These include negative messages about power, economic independence, vocational goals, physical attractiveness, sexuality, and family roles. When disability is also part of the picture, the situation is even more complex for the woman.

Hanna and Rogovsky (1992) have identified a number of factors that operate in the double discrimination of being a woman and having a disability. The first relates to nurturing roles. Women are traditionally thought of as being the nurturers in our society, that of wife, mother, sexual partner, and the superwoman office worker. Women with disabilities are often viewed as being unable to engage in sexual activity, as being incapable of motherhood, and lucky if they ever find a partner (Alston \& McCowan, 1994; Hanna \& Rogovsky, 1992). They receive the message they will be unfit mothers, that they are dependent people who must themselves be nurtured. Physical attractiveness is an influential element in determining a person's worth, especially for women. According to Danek (1992), as long as society imposes unrealistic body image 
expectations, women with disabilities will continue to be sexually and personally devalued. A study by Hanna and Rogovsky (1991, cited in Danek, 1992) found that the word "woman" was often connected with terms that had connotations of beauty. When the adjective "disability" was added to "woman", the phrase was frequently associated with such words as "ugly" or "unpleasant."

People often hold separate prevailing beliefs about the causes of disabilities for men and women (Hanna \& Rogovsky, 1992). Many attribute the disabilities of males as being related to relatively postive external factors such as war/fighting and work. Beliefs concerning the causes of disabilities for females are more negative. They are more likely to focus on internal traits of the individual, on "imperfections" such as carelessness and illness. The U.S. Bureau of the Census (1989) reports 33 percent of women with disabilities are employed compared to 69 percent of men with disabilities. Of this number, only 13 percent of the women have full-time jobs.

Fine and Asch (1981, cited in Danek, 1992) have used the tern rolelessness to describe women with disabilities who fill neither the traditional nurturing role nor that of the economically independent worker. The absence of productive roles can lower self-esteem and may be demoralizing (Danek, 1992). Danek concludes that many 
women with disabilities have not yet experienced "the personal empowerment that comes from full participation in both the mundane and the extraordinary that comprise the daily life of most citizens in the 1990s" (p. 11). Other women with disabilities find the challenge of overcoming social and environmental barriers too overwhelming. This perpetuates the relative isolation from social contacts and the workplace, inadequate education, low income, lack of resources, and dependence (Hanna \& Rogovsky, 1992).

\section{Culture}

Each individual is a part of a culture and a society, elements of each helping to define who that person is (Cassel, 1982). Culture determines the meanings of masculinity and femininity, acceptable mating behavior, appropriate dress, beliefs toward the sick and dying, and the response and treatment of the aged and the disabled. Culture and society regulate whether the sick and disabled will be accepted or be isolated and whether they will be received with compassion or ignored. Culture also has a large influence on the manner in which loss is perceived and experienced (Osterweis et al., 1984). It sanctions the categories and norms defining the consequences of loss, determines how stressful the loss is relative to other stressors, what is expected concerning social support and personal coping styles, and preferred ways of regarding and 
responding to death. Social class, economic status, and sociopolitical factors contribute to variability of individual reactions and deviation away from group norms. As a result, there are likely to be both significant continuity and notable variation to the experience of loss across cultures, ethnic groups, and social classes. Viewing loss strictly according to Western standards is indicative of an insensitivity to the cultural influences contributing to the ways individuals and families grieve. Western society endorses youth, vitality, and efficiency, loss and grief often represented as obstacles to a smoothly running system (Hendrick, 1981). Admiration is expressed for the "strong and able" and a subtle negativity toward the "weak and ill" (p. 361). Grieving behavior is appropriate but only if it does not last too long. Grief is seen as a problem, an emotional state that lasts for a period of time and must be endured (Parry, 1994). Western cultures have abandoned many of the rituals, the "rites of passage" that serve basic human needs in periods of transition (Parkes, 1985).

Stroebe (1992-93) provides evidence from other cultures countering the widely held Western belief that one must work through grief. The Navajo people are limited to a four day mourning period, after which they are expected to return to normal life. It is considered beneficial to suppress loss rather than confront it. Likewise, the 
Samoan people recover rapidly and comparatively painlessly Ablon (1971, cited in Stroebe) attributes this to differences in the value systems of these cultures, the principle of reciprocity being the key in the easy transition through bereavement. In the two Muslim societies of the Balinese and the Egyptians, grief work is encouraged in the latter society, but looked down upon in the former. The Japanese people do not try to achieve detachment from a deceased individual. Rather "grief work" functions to retain the relationship and bond with the deceased. In some Eastern cultures, death is often seen as a beginning and a release from earthly distress (Parry, 1994 ). Death is a highly revered experience consisting of extensive and highly structured rituals, usually lasting over a period of weeks. There may also be observances on specific dates during the year. Rituals are a resource for mouning death and other losses (Davis, 1987). They emphasize story telling, traditional wisdom, sharing of food, and give permission to express overwhelming feelings by laughing, crying, or singing.

Sue and Sue (1990) define world view as the attitudes, values, and concepts that affect how people think, make decisions, behave, and define events. World views also influence cognitive processes including communication styles, information processing, and social interactions (Sodowsky, Maguire, Johnson, Ngumba, \& Kohles, 1994). A 
number of categories have been suggested to increase the understanding of the differences among individuals and groups. They are the nature of people, time focus, social relations, attitudes toward activity, and the relationship between people and nature. (Sue \& Sue, 1990). An individual who values independence, autonomy, and personal control, may react differently to loss or disability than a person who prioritizes group goals over individual goals and has a fatalistic outlook on life.

Some members of American minority groups may have few resources for carrying out their culturally expected bereavement practices but have not yet adopted the dominant culture practices (Osterweis et al. 1984). SoutheastAsians may question the use of funeral homes as a way of dealing with death and have no cultural conceptualization of professional grief counseling. The Indochinese focus on interdependence rather than personal choice and independence (Sue \& Sue, 1990). They believe in living in harmony with nature, viewing mental illness as resulting from an imbalance in cosmic forces or lack of will power. Mental illness is also believed to be a failure of the family. Physical problems are seen as causing emotional disturbances which will disappear as soon as the physical illness is successfully treated. Physical complaints are a commonly used and a culturally accepted way to express psychological and emotional stress. 
Smart and Smart (1991) identify five factors they believe are associated with the acceptance of disabilities among Mexican Americans. The first is a familiar, cohesive, protective society. The Hispanic family unit is a problem solving unit, serving as a retreat from the outside world (Smart \& Smart, 1994). It is characterized by deep mutual obligations and intense relationships. Hispanic families tend to shelter and paternalize their disabled, especially children. Encouragement of independence seems to correspond with increases in age and socioeconomic status of the disabled person. Illnesses and disabilities may become extensions of the person with family loyalties and other cultural factors such as religion promoting dependency and isolation (Garza, 1986, cited in Smart \& Smart, 1991).

The second factor is the stoic attitudes toward life in general. A common feeling is that life is hard because of unavoidable hardships and suffering and should be endured with dignity and courage. Illness is seen as a sign of weakness of character, the need for treatment viewed as disgraceful and may result in a loss of pride. The third factor influencing acceptance of disability is the presence of well-defined gender roles. Hispanic women are taught to be homemakers and the men to be providers for the family. Disability may have even a greater impact on the male's self-image, his life devastated when he can no 
longer fill his role. The fourth factor identified by Smart and Smart (1991) is religious views. Disabilities are often believed to have a supernatural etiology. It may be interpreted as divine punishment for sin. Such interpretations may led to the assumptions that Hispanics are fatalistic and have an external locus of control. Garza (1986) contends that this view is inaccurate because most researchers have little understanding of the cultural dynamics of the Chicano community. The final factor is the reliance on physical labor. Many Hispanics have lower levels of education and rely principally on physically demanding jobs. Smart and Smart (1991) express the opinion that acceptance of disability is related to the number of available resources and options. Onset of disability may limit employment and training opportunities. Disability may not only represent loss of physical ability but loss of status.

African Americans with disabilities face the double bias of being a racial minority and of having a physical impairment (Alston \& Mngadi, 1992). As a group, African Americans are often unemployed or underemployed and undereducated. It has been suggested that the African American family has developed unique patterns of competence which serve as buffers to the stressors and crises commonly faced by its members (Alston \& McCowan, 1995). These buffers are used to manage typical crises faced by most 
families such as divorce, death, or disability. In their study exploring family competence factors on adjustment to disabilities for African Americans, Alston and McCowan (1995) found that African American who adjust to their disabilities come from close and supportive families with feelings openly shared both verbally and physically. They interviewed 64 African Americans in a Southeastern state whose cases had been successfully closed by the Department of Vocational Rehabilitation and the Department of the Blind, exploring what impact factors such as cohesion, expressiveness, leadership, and conflict have on adjustment to disability. Alston and McCowan (1995) concluded that a sense of family well being was a key contributor to psychosocial adaptation. African American families can accommodate disability and continue to carry on a healthy balance of behaviors such as humor, stability, and minimal preoccupation with selfish issues.

Alston and McCowan (1995) also found relationship between adjustment and leadership patterns. According to Alston and McCowan, African Americans tend to ignore rigidly prescribed roles in society which has resulted in the absence of clearly defined leadership in the family. Men, women, and children are permitted to develop multiple roles in the family (Sue \& Sue, 1990). The leadership role traditionally assigned to the father may be shared by several members in the African American family (Alston \& 
McCowan, 1995). This flexibility in role redefinition may serve to facilitate adjustment when a family member incurs a disability. Alston and McCowan (1995) believe cohesion, the existence of a close family unit, is the strongest predictor of adaptation to disability in African Americans. They view the strength and resourcefulness of the African American family as being crucial to successful psychosocial adaptation to disability .

American Indians believe in a holistic view of health in which the mind, body, spirit, and nature are one harmonic process (Braswell \& Wong, 1994). Illness or disability is seen as a disruption of this harmony with medicine and religion being a major part of the healing process. Often, the shaman or medicine man/woman takes the role of physician, psychiatrist, and spiritual leader using rituals, herbs, and ceremony to restore the balance between the natural and spirit world. Despite the great diversity among tribes, there are some generalizations that can be made concern American Indian values (Sue \& Sue, 1990). While status is gained by accumulation of goods in the dominant culture, Indians earn respect and honor by sharing. Indians hold the belief that family and group are more important than the individual, emphasizing interrelationships between a large number of relatives. They are taught to observe rather than act impulsively and not to interfere with others. Focus of time is very much 
on the present rather than on the future. There is a strong respect for the wisdom and knowledge of elders. Rather that attempting to master the environment as the dominant culture does, Indians accept things as they are.

Osterweis et al. (1984) caution against stereotyping behaviors of any group of people in an effort to be culturally sensitive. It is essential to take into account how much acculturation and social mobility have altered beliefs and behaviors along with the wide range of intraethnic diversity. Education, socioeconomic background, and family experiences may also impact each person in a different way (Sue \& Sue, 1990). Knowledge of cultural values can be used to help form conceptions on how a minority might view a disorder and expectations of treatment, but must not be applied in a rigid manner.

\section{Variables Specific to Disability}

The last section of this chapter will discuss variables that might influence reaction to loss that relate specifically to disability. Time of onset has already been discussed, viewing it from a life-span perspective. More needs to be said about whether the disability was present at birth or whether it happened later. The individual with a congenital disability may be subjected to isolation, may be overprotected or rejected as a child, and may be separated from the mainstream in regards to family life, 
play, and education (Vash, 1981). Although the person experiencing disability later on in life may not have to face these issues, there will be others that must be dealt with.

Research by Jabaley, Hoopes, Knorr, \& Meyer (1970, cited in Vash, 1981) revealed that children with visible disabilities do not begin to realize they are different from other children before they reach the age of four. Children who have been overly protected by their parents may be devastated when they eventually are subjected to ridicule or rejection from their peers (Marshak \& Seligman, 1993). Their increased awareness of their disability may be as traumatic as if they were suddenly injured. The child may not undergo shock, but might experience a period of mourning including denial of their condition. Adolescents are under tremendous pressure to conform to norms of society (Wright, 1983). Loss may be experienced in terms of personal and social satisfactions which are now believed to be denied to them. Much like the chronic sorrow concept presented by Burke and her associates (1992), there may be grieving over the loss of possibilities associated with a nondisabled state - the life that could have been led (Marshak \& Seligman, 1993), Type of disability is another factor that may influence reactions to disablement. Each disability, whether it be losing eyesight, hearing, or the ability to 
walk will involve different reactions since each creates different problems (Vash, 1981). Much of the impact of the specific impairment is closely related to the characteristics of the disabled individual. A musician might suffer greater devastation from loss of hearing than from loss of sight. Shontz (1977) makes it clear that knowledge of the type of a person's disability provides virtually no information about his or her personality. It is not unusual for an individual to show increased body anxiety and depression with onset of disability. However, these responses often do not exceed what is expected of an equally strong psychological stressor. For example, the death of a loved one may bring on similar reactions. The degree to which the disability interferes with physical attractiveness could also contribute to reactions (Vash, 1981). This might be especially true in societies where significant value is placed on how a person looks. Another aspect is visibility of disability (Vash, 1981). Invisible disabilities can be difficult, because the individual does not appear to be what he or she actually is. A large, muscular man with chronic back problems may grow tired of having to explain why he cannot help move heavy objects. Stability of the disability is dependent on how much the disability changes over a period of time. A condition characterized by an unpredictable course may invoke different reactions than a disability 
that occurs suddenly, shows some improvement, and reaches a stable plateau. Periods of remission may be accompanied by feelings of hope only to be replaced by disappointment, fear, and anger as symptoms reappear. People with progressive disabilities are subject to the uncertainty of the nature of the disorder and to the uncertainty of their future.

Severity of disability is another variable that could influence response to disability. It is not the extent of loss alone that determines reaction, but rather it is the ecological fit between the person and his or her environment that determines behavior (Lilliston, 1985). Adjustment to loss is influenced by coping strategies, family support, financial resources, internal psychological factors such as creativity and preference for interpersonal support. Frey (1992) conducted a study which showed that severity of injury is not related to the length of hospital stay. He concluded that psychological variables play a much greater role in the recovery process, overriding the severity of injury. Wright (1983) speculated that persons with mild disabilities may try harder to hide and deny the disability because they are almost normal. The presence of pain is also certain to influence the person's feelings and behaviors (Vash, 1981). The degree to which pain predominates is largely dependent on past experience. 
Both the immediate and the broader cultural aspects of the environment have powerful influences on emotional and behavioral reactions to disability (Vash, 1981). This relates to family support, income, available community resources, the degree of technology to resolve functional problems, and funding to protect the rights of people with disabilities. Architectural barriers impose limitations on mobility, education and vocational opportunities, and on interpersonal adjustment (Shontz, 1977). Such barriers send the message to people with disabilities that they are inferior and will be kept that way. With constant exposure to the negative attitudes of society, it becomes increasingly difficult for the individual to see himself or herself as competent and worthwhile.

Before ending this chapter, it is important to stress that despite having a disability a satisfying life is possible and in most cases probable. Although many researchers have tried to correlate the onset of disability with maladaptive personality attributes, the evidence shows that psychiatric disturbances in reaction to disability do not, in general, differ from what is the considered normal rate of psychological distress in the general population (Shontz, 1977). Although finding life more difficult, most people with disabilities report their levels of happiness, satisfaction, and self-esteem as being on a par with the non-disabled population (Marshak \& Seligman, 1993). With 
the passage of time, people adapt to major life events, the events gradually losing their ability to evoke strong emotions. Living with a disability is not seen as a tragic event, but a fact of life and/or an inconvenience. Disability may not necessarily be the most important thing in a person's life (Shontz, 1977). 


\section{CHAPTER V}

CHOOSING A THEORY OF LOSS

The Attributes of a Good Theory

In Chapter 3, a number of different viewpoints were presented that offer explanations of reactions to loss. Special attention was paid to the losses experienced after onset of disability. Chapter 4 focused on the factors that might influence individual response to loss. These included such variables as age, gender, culture, personality traits, the quality of support systems, environmental conditions, and differences relative to type and degree of disability. This leaves the question of what theory is the most appropriate for the individual suffering the loss. According to McWhirter and McWhirter (1991), theories serve as guides to making some kind of sense out of life, providing consistency and unity. Beyond this, they see theories as organizing information and data into more usable, more understandable, and more useful forms. No single theory is perfect and there are no guarantees against misuse of existing models (Corr, 199192; Shontz, 1980). At times, different theories are valid under different circumstances (Shontz, 1980). Theories 
evolve in response to the needs of people, needs that are constantly changing. Old theories become outdated as new problems arise, calling for new viewpoints and new solutions. Corr (1991-92) stresses that it is not enough to just criticize these theories or just to set them aside. Instead, it is essential to develop better models, presenting them in ways to minimize the chances of being misunderstood. It has been the intent of this paper to look at a cross section of the literature on loss, past and present, to provide some direction in working with people with disabilities. Ultimately, this would result in the helper applying theory and methods more effectively.

To help reach this goal, it is valuable to understand what makes a good theory. Shontz (1981) has elaborated on the work of Meyerson listing six characteristics of good theories. First, a good theory is a generalization, but is also valid when applied to the individuals. Ideally, it pertains to all people accounting for exceptions within the assumptions of the theory. Second, a good theory makes the least possible number of assumptions, fewest concepts, and offers minimal mechanisms for explaining behavior. This guards against the tendency to confuse labels with explanations. The third characteristic of a good theory is that it explains why and how particular events arise from particular causes. It stresses functional relationships 
rather than associations. Fourth, a good theory expedites decision making, stimulating the growth of practical plans of action. Fifth, a good theory is dynamic, describing forces that bring about observed states or relations. It goes beyond superficial appearances by trying to find underlying causes. The focus is on what is happening now and not what occurred in the distant past. The final characteristic on Meyerson's list is that a good theory stimulates research. Good theories do not give all the answers but often contribute by acknowledging their shortcomings, suggesting topics for further research. These are all points to consider when looking at the various theories of loss.

Another area to consider is whether the theories of loss, originally applied to lost of a loved one, are relevant to the losses of the person with a disability. Reich, Zautra, and Guarnaccia (1989) present evidence showing significant differences between the two. Their data suggest that loss from disability may have a greater adverse, long-term effect than from loss of a significant relationship. The bereaved subjects were more depressed, but were less anxious. The disabled group demonstrated lower positive well-being and greater distress than the other subjects in the study. Although the disabled subjects continued to show considerable psychological 
distress over time, the bereaved subjects showed recovery on several aspects of mental health. The bereaved group improved, manifesting increased positive affect and less depression and helplessness/hopelessness. Reich et al. (1989) offer two reasons for the discrepancy. One, the disabled subjects continue to experience the effects of the stressor on a daily basis. Second, they believe the effects of disability on mental health are greater, influencing both self-concept and emotional states. It may take longer to repair damage to self-cognitions than those situations which only influence affective states. While this study focused on older adults, it brings up the question of how widespread a phenomenon this is.

Advocates of chronic sorrow veer from the prevalent model of linear, time-bound grief following the death of a loved one. Davis (1987) contends people with permanent disabilities, chronic illnesses, degenerative conditions, and mental retardation or mental illness experience grief differently. Their recurrent mourning requires that they come to terms with a changed self. It is a life-changing process because the sorrow becomes a part of the person's life and because of strong pressures from society to deny its presence or to cope heroically. The sadness of chronic sorrow is permanent, varies in intensity between situations and persons, and is interwoven with periods of neutrality, 
satisfaction, and happiness (Teel, 1991). In chronic sorrow, the ongoing presence of the disability prohibits resolution of the loss. Kübler-Ross (1974) states it is sometimes more difficult and requires more time to accept disability than to face death, "which is at least an end to the suffering "(p. 27).

Arguments for an Integrated Theory of Loss

There is no single theory that is universally correct - that can universally explain all facets of human behavior (McWhirter \& McWhirter, 1991). Moreover, some theoretical approaches are more effective and directly applicable for presenting problems than other approaches. McWhirter and MCWhirter (1991) suggest using several existing theoretical viewpoints in the hopes of increasing the helper's ability to apply theory and methods more effectively. They define eclecticism as "the selection by the helper of an approach that meets the needs of the client and that is particularly adaptable to a problem or a situation" (p. 77). Corey (1991) describes eclectic counseling as the process of choosing concepts and methods from a variety of systems. According to the American Heritage Dictionary (1980), integrate is defined as choosing what appears to be the best from diverse sources, systems, or stages. Corey (1991) uses the term integrationism in reference to a 
"conceptual or theoretical creation beyond a blending of techniques" (p. 426).

Wortman and Silver (1987) have suggested that there are at least three different patterns of adaptation to loss. There are some individuals who go through the expected pattern, moving form high to low distress and resolving their grief over time. Others remain in a distressed state beyond the time frame recognized as normal by traditional theories of grief. A third group appears to show little or none of the expected distress immediately after a loss or at subsequent intervals. Wortman and Silver (1987) believe that traditional views about what is a "normal" response to loss have impeded theoretical research in this area. Theories focusing on a universal process of grief provide little insight accounting for variations in response to loss.

MCWhirter and MCWhirter (1991) suggest that counselors examine aspects about themselves that create a personal attraction for a specific theory. At the same time, it is equally important to examine neutral or negative responses toward other theories. According to McWhirter and McWhirter, this continual, active process leads to increased self-knowledge and to a better understanding and application of theory. As mentioned above, Corr (1991-92) contends it is necessary to go beyond criticizing and 
ignoring theory to improve analyses of grief and mourning. Although Wortman and Silver (1987) have made some valid points concerning traditional theories of loss and grief, they have not acknowledged the contributions made by these stances. Despite their shortcomings, there may indeed be something that may be learned form them. Completely disregarding theories such as the stage-based approach fails to take into account the people who do seem to go through loss in a linear progression.

Marshak and Seligman (1993) have found themselves alternately drawn to and repelled by the different aspects of the various perspectives on adjustment to disability. They draw from many different theoretical orientations in order to address the emotional, cognitive, and behavioral aspects of adjustment. Briefly looking back at some of the theories discussed, each has made at least one contribution in laying the groundwork for theories of loss and grief. without them, the field may not be where it is today.

Although psychoanalysis is often criticized for its outlook on ego defects and feelings of inferiority, it has offered concepts still actively used today. Defense mechanisms are often used to describe reactions to disability. The concept of Thanatos, or the death instinct, may offer a partial explanation for selfdestructive behaviors that eventually result in physical 
disability (Cook, 1992). According to Cubbage \& Thomas (1989), Freud was the first person to apply the concept of secondary gain to persons with disability.

Kübler-Ross (1969) was neither the first person nor the last one to use a stage-based model for understanding coping with dying. Many people question the validity of her work, the arguments against using stage theories being presented in Chapter 3. A major concern is that such models risk stereotyping vulnerable individuals who are coping with dying and loss (Corr, 1991-92). Although Corr (1993) believes the stage-based model of Kübler-Ross is inherently inadequate, he points out there is much that can and should be learned from her. She was an early leader and important publicist in the campaign to increase understanding of coping with dying. He credits Kübler-Ross with helping to bring about significant changes in the way in which people envision dying at a time when there had been so much distancing from the subject. She showed great sensitivity in her observations of dying patients, focusing on them as human beings.

Kübler-Ross (1969) looked at the dying patients as teachers, enabling others to learn about the final stages of life with its anxieties, fears and hopes. By drawing from the experience of the dying, we as observers can come to know ourselves better. Kübler-Ross represented coping 
with dying as a human and vital process, a time when there is often unfinished needs the individual may want to address. She emphasized the necessity to actively listen to the needs and priorities of the dying person in order to be or become an effective provider. The work of KuiblerRoss is a reminder that dying patients are still livinghuman beings.

These are some examples of positive aspects of two theoretical approaches that might be integrated into a holistic perspective on loss and grief. Corey (1991) contends that developing an integrated theoretical perspective is a challenge, requiring much reading, thinking, and counseling experience. He believes it is essential to have an accurate and in-depth knowledge of theories to be able to form a true synthesis. In making choices, it is important to know the original intentions of the person developing the theory to avoid misuse and confusion. The writing of kübler-Ross has been interpreted, reinterpreted, and finally distorted to the point where it no longer meant what she originally intended it to say. It is the responsibility of the individual to verify his or her selections, ideally consulting the original source. A true integrated approach involves deliberate choices, incorporating terminology, techniques, concepts, and procedures to form one unified theory 
(McWhirter \& McWhirter, 1991). It is through such an endeavor, that the helper is better able to assist the individual who is recovering form loss.

An integrated approach to loss involves not only combining features from different theoretical perspectives but also includes both personal factors and environmental factors (Russell, 1981). Personal factors focus primarily on what happens to the person and is concerned with behavior and with the mental aspects of adjustment. The environment consists of the social and physical conditions confronting the person. Adaptation to loss occurs through the interaction between the individual and his or her environment. The integrative approach of Moos and Tsu (1977) takes into account background and personal factors, disability-related factors, and physical and social environmental factors. These factors interact with each other and determine the course and outcome of the crisis. The background and personal factors include age, intelligence, cognitive and emotional development, philosophical or religious beliefs, and previous coping experiences. They influence the ways in which the individual perceives the meaning of disability, the kinds of adaptive tasks that arise from the disability, and what coping skills are used. 
Disability-related factors include the type and location of symptoms. Considerations are whether the illness or disability is painful, disfiguring, disabling, and the special importance the affected body region has to the individual. To illustrate this last point, a woman in early adulthood may experience greater psychological impact from the loss of reproductive organs than an elderly woman. Physical and social environmental features, the third set of factors, can either contribute to the stress or be sources of help and support. Physical factors may include conditions in the hospital or in the person's home, the esthetic quality of one's surrounding, the amount of personal space available, and the degree of accessibility in the community. The social environment includes the relationship between the individual and his or her family, social supports in the community (such as friends, associates, clergy, and social service agencies, and sociocultural norms and expectations. According to Moos and Tsu (1977), the extent to which family members are able to support the person with the illness or disability and each other strongly affects the choice of coping strategies and the eventual outcome. 
An Integrated Model of Loss

The model of Moos and Tsu (1977) contributes to a better understanding of the crisis of illness or disability. It stresses the importance of integrating the personality of the individual, the nature of the disease or injury, and the unique set of environmental circumstances in determining the outcome of a crisis. While Moos and Tsu offer an explanation why people might respond differently to illness and disability, they do not include a process of acceptance or adaptation in their model. I am proposing a model that incorporates key points of many of the theories that have been presented in this thesis.

This model is based on the assumption that grief is a unique process which may be positively or adversely influenced by environmental and emotional factors and by the world of the individual. The model tries to account for the variable responses to loss observed by Wortman and Silver $(1987,1989,1992)$. It contends that there is not one theory that adequately argues for a universal adaptation to loss or explains why people react differently to loss. Rather, due to the many possible environmental, cultural, and psychological factors, the unique attributes of the individual, and to the of the nature of the loss itself, any combination of loss theories could be in effect for any one loss. This integrated model does not suggest 
that progressing through stages or phases is a necessary part of the grieving process. Rather it suggests there may be multiple reactions to loss one of which may be a linear progression. For example, an individual with a spinal cord injury might first progress through stages to achieve adaptation to the loss. As other losses associated with the disability occur (loss of mobility, autonomy, income, etc.), other theories such as chronic sorrow (Burke et al. 1992; Davis, 1987; Teel, 1991) or Wright's (1983) value change theory may account for adaptation to the loss. The bottom circle of the model (see Figure 1) represents the person and the multiple factors that together form a state of physiological, social and psychological equilibrium in his or her life. A disruption or change in any area of the circle impacts other areas. A crisis, such as disability, offsets the balance and is a threat to the total well-being of the individual. According to crisis theory, a person in a state of extreme disequilibrium is self-limited (Moos \& Tsu, 1977). This state of disorganization is often characterized by such feelings as anxiety, fear, and guilt which lead to further disorganization. Resolution requires that the individual reestablish a new balance. A healthy adaptation to crisis is one that creates personal growth while a maladaptive response results in psychological maladjustment. 


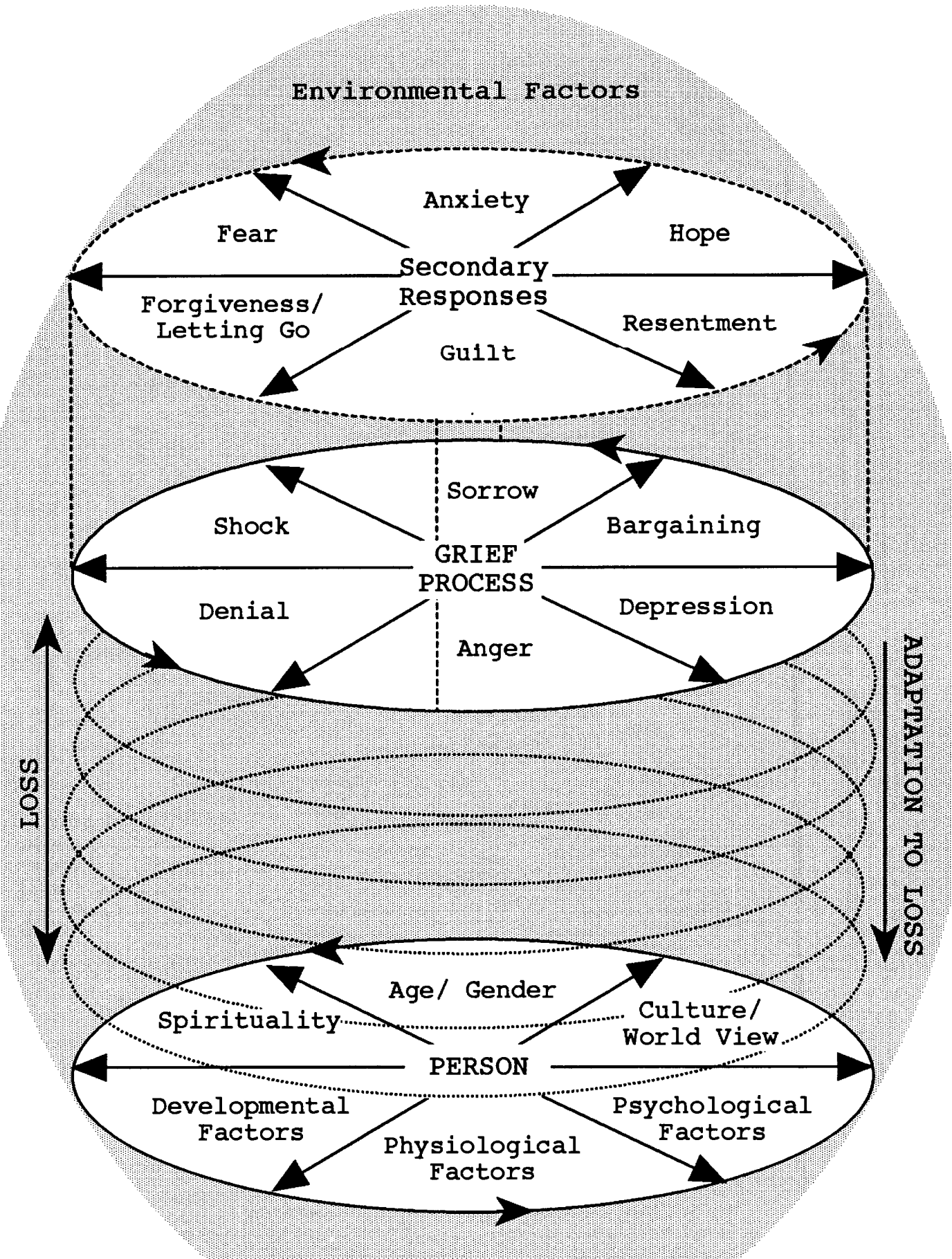

Environmenta 1 Factors

Figure 1. An integrated model of loss 
The grief process is patterned after the figure-ground concept which is an integral part of Gestalt theory. Gestalt theory hypothesizes that the figure represents the individual's most urgent need and appears in the foreground (Grossman, 1989). Once the need is satisfied, the figure fades into the background allowing for the next need to be brought to the foreground. This process is impeded when needs are not recognized or expressed and remain in the background. These unmet needs not only require energy to maintain but also interfere with new figure formation. The figure-ground concept can be applied to loss and to the phases of the grief process. The needs of the individuals are those that are essential for successful adaptation to the loss. The needs will vary from person to person, largely dependent on the factors identified in the person section of this integrated model of loss. A child who has been disabled since birth may not experience a disability-related loss while in a protective family environment. As the child matures physically and developmentally and becomes exposed to a school atmosphere, losses may begin to occur. The child may begin to recognize he or she is different from other children, becoming increasingly aware of physical or cognitive limitations. He or she may be ridiculed by peers or excluded from group activities. A shock phase would be 
rare in these circumstances but the child may initially experience sadness (depression), denial or anger. Conversely, the young adult who suddenly finds himself or herself paralyzed is more likely to undergo an initial phase of shock before experiencing other phases of the grief process.

This integrated model of loss is based on the assumption that there is no set order of proceeding through the grief process. Furthermore, the process may last for different periods of time, may reoccur at a later time, and may exist side-by-side. What is important is that there is always one dominant phase that requires the primary attention and energy of the person. As the needs of this phase are being met, another is already coming to the surface to eventually replace the primary phase. The secondary responses are experienced concurrently with the phases of the grief process. These are not the only responses to loss but represent some of the most frequent reactions to loss. Unlike the movement through the grief process during which one phase predominates, any number of the secondary responses may be present at the same time. When, how long, and the combination of the responses is unique to the person and to the circumstances of the loss. Kuibler-Ross (1969) has suggested that hope often exists throughout all the stages of her model of loss. 
Some people may experience all the phases while others may only have to undergo one or two to achieve adaptation to the loss. Others, as Wortman and Silver (1987) have suggested, may not need to go through this entire grief process. This group of individuals is able to return to a state of equilibrium with minimal effort. The integrated model can also be used to illustrate chronic sorrow. Individuals faced with ongoing reminders of their loss might go through the entire cycle of loss, the grief process, and adaptation, many times during the course of their illness or disability. As the person deals with the loss, equilibrium is reestablished. The balance may once again be upset as events occur that set off the grief process all over again. A child may realize that he or she cannot physically compete with his or her peers or a marriage may end due to illness or disability related factors. Individuals who are not able to progress through the grief process may remain in a constant state of sorrow. Dysfunction occurs when the person is unable to proceed through the necessary phases for adaptation and becomes "stuck" in the process of recovery. An example of this would be a previously very active individual who is faced with a progressive disease such as multiple sclerosis. If this person continues to place great value on physical activity, he or she could remain in the grief 
process indefinitely, especially as symptoms become progressively worse. This corresponds closely to the concepts of Wright (1983), which emphasize the necessity of changing the value structure of the person to achieve acceptance of loss.

Adaptation is achieved by establishing a new balance. The loss is not forgotten but integrated into the whole of the person. Weenolsen (1992) and Vash (1981) refer to this process as transcendence. Horowitz (1983) refers to it as completion tendency, which occurs once the mind processes the new information and accepts the new reality. According to Cohn-Kerr (1961), adjustment takes place when the individual no longer thinks of disability as a barrier to be fought. Instead, the disability becomes one of the characteristics of the person along with his or her other assets and liabilities.

It is important to realize that this model of an integrated model of loss is only an initial attempt to integrate data and perspectives on the subject of loss and needs to be validated by research. This paper has not identified any single approach as being superior in understanding adaptation to loss and disability. It has stressed the importance of recognizing the numerous forms and levels of adaptation. Hopefully, the readers will see the advantage of an integrated approach. The intent is to 
encourage a holistic outlook on the subject of loss which will lead to selecting the most appropriate intervention for the particular client and his or her specific situation. 
REFERENCES

Ahmed, P. I. (1992). Living and dying with AIDS. New York: Plenum Press.

Ablon, J. (1971) . Bereavement in a Samoan community. British Journal of Medical Psychology, $44,329-337$.

Alston, R. J., \& McCowan, C. J. (1994). African American women with disabilities: Rehabilitation issues and concerns. Journal of Rehabilitation, 60(1), 36-40.

Alston, R. J., \& McCowan, C. J. (1995). Perception of family competence and adaptation to illness among African Americans with disabilities. Journal of Rehabilitation, 61(1), 27-32.

Alston, R. J., \& Mngadi, S. (1992). The interaction between disability status and the African American experience: Implications for rehabilitation counseling. Journal of Applied Rehabilitation Counseling, $2 \underline{3}(2), 12-16$.

Ansbacher, H. L. (1980). Theories of personality and psychopathology: Cultural and interpersonal psychoanalytic schools. In H. Kaplan, A. Freedman, \& B. Sadock (Eds.), Comprehensive textbook of psychiatry III (pp. 729-740). Baltimore, MD: Williams \& Wilkins Company.

Baker, E. (1985). Psychoanalysis and psychoanalytic psychotherapy. In S. Lynn, \& J. Garske (Eds.) Contemporary psychotherapies: Models and methods. Columbus $\mathrm{OH}$ : Charles Merrill Publishing Co.

Becker, E. (1973). The denial of death. New York: The Free Press, Macmillan Publishing.

Blacher, R. S. (1970). Reaction to chronic illness. In B. Schoenberg, A. Carr. D. Peretz, \& A. Kutscher (Eds.), Loss and grief: Psychological management in medical practice (pp. 189-198) . New York: Columbia University Press. 
Blumberg, B. D., Lewis, M. J., \& Susman, E. J. (1984). Adolescence: A time of transition. In M. Eisenberg, L. Sutkin, \& M. Jansen (Eds.). Chronic illness and disability through the life span (pp. 133-149) . New York: Springer Publishing Company.

Bowlby, J. (1961). Process of mourning. International Journal of Psychoanalysis, $\underline{42}$, (parts $3 \& 4$ ), 317-340.

Bowlby, J. (1973) . Attachment and loss: Vol. 2 . Separation: Anxiety and anger. New York: Basic Books.

Bowlby, J. (1963). Pathological mourning and childhood mourning. Journal of the American Psychoanalytic Association, $11,500-541$.

Brandtstädter, J., \& Renner, G. (1992). Coping with discrepancies between aspirations and achievements in adult development: $A$ dual-process model. In $\mathrm{L}$. Montada, S. Flilipp, \& M. Lerner (Eds.), Life crises and experiences of loss in adulthood (pp. 301-319). Hillsdale, NJ: Lawrence Erlbaum Associates.

Braswell, M. E., \& Wong, H. D. (1994). Perceptions of rehabilitation counselors regarding Native American healing practices. Journal of Rehabilitation, 60(2), 33-37.

Breger, L. (1974) . From instinct to identity: The development of personality. Englewood Cliffs, NJ: Prentice-Hall.

Bretherton, I. (1991). The roots and growing points of attachment theory. In C. Parkes, J. Stevenson-Hinde, \& P. Marris (Eds.), Attachment across the life cycle (pp. 9-32) . New York: Routledge.

Bretherton, I. (1992) - The origins of attachment theory: John Bowlby and Mary Ainsworth. Developmental Psychology, 28 (5), 759-775.

Broden, A. R. (1970). Reaction to loss in the aged. In B. Schoenberg, A. Carr. D. Peretz, \& A. Kutscher (Eds.), Loss and grief: Psychological management in medical practice (pp. 199-217). New York: Columbia University Press. 
Burke, M. L., Hainsworth, M. A., Eakes, G. G., \& Lindgren, C. L. (1992) . Current knowledge and research on chronic sorrow: A foundation for inquiry. Death Studies, 16(3), 231-245.

Cannon, W. B. (1939), The wisdom of the body. New York: Norton.

Carnes, G. D. (1967). Personality abnormalities among patients on a physical medicine and rehabilitation service. Archives of Physical Medicine \& Rehabilitation, $\underline{45}, 321-325$.

Cassel, E. J. (1982). The nature of suffering and the goals of medicine. The New England Journal of Medicine, 306 (11), 639-645).

Cassem, N. H. (1975). Bereavement as indispensable for growth. In B. Schoenberg, I. Gerber, A. Wiener, A. Kutscher, D. Peretz, A. Carr (Eds.), Bereavement: Its psychosocial aspects (pp. 9-17). New York: Columbia University Press.

Cheikin, M. L. (1981). Loss and reality. The Personal and Guidance Journal, $\underline{59}(6), 335-338$.

Cohn-Kerr, N. (1961). Understanding the process of adjustment to disability. Journal of Rehabilitation, $\underline{27}(6), 16-18$.

Cook, D. (1992). Psychosocial impact of disability. In R. Parker \& E. Szymanski (Eds.), Rehabilitation counseling: Basics and beyond (pp. 249-272). Austin, TX: PrO-Ed.

Corey, G. (1991). Theory and practice of counseling and psychotherapy. Pacific Grove, CA: Brook/Cole.

Corr, C. A. (1991-92). A task-based approach to coping with dying. Omega, 24 (2), 81-94.

Corr, C. A. (1993). Coping with dying: Lessons that we should and should not learn from the work of Elisabeth Kübler-Ross. Death Studies, 17, 69-83.

Cubbage, M. E., \& Thomas, K. R. (1989). Freud and disability. Rehabilitation Psychology, 34(3), 161173. 
Cytrynbaum, S., \& Patrick, R. (1979). Mid-life development from a systems perspective. In S. Cytrynbaum (Chair), Midlife development: Influence of gender, personality, and social systems. Symposium presented at the meeting of the American Psychological Association, New York.

Danek, M. M. (1992). The status of women with disabilities revisited. Journal of Applied Rehabilitation Counseling, $\underline{23(4), 7-13}$.

Darwin, C. (1965). The expression of the emotions in man and animals. Chicago: The University of Chicago Press. (Originally published 1872).

Davis, B. H. (1987). Disability and grief. Social Casework, 68 $(6), 352-357$.

Davis, S. E., Anderson, C., Linkowski, D. C., Berger, K., \& Feinstein C. F. (1985). Developmental tasks and transitions of adolescents with chronic illness and disability. Rehabilitation Counseling Bulletin, 29 (2), 69-80.

Dembo T., Leviton, G. L., \& Wright, B. A. (1956). Adjustment to misfortune: A problem of socialpsychological rehabilitation. Rehabilitation Psychology, 22, 1-100.

Demi, A. S., \& Miles, M. S. (1986). Bereavement. In H. Werley, J. Fitzpatrick, \& R. Taunton (Eds.), Annual review of nursing research (Vol. 4, pp. 105-123). New York: Springer.

Dohrenwend. B. S. (1978). Social stress and community psychology - American Journal of Community Psychology, $\underline{6}, 1-14$.

Dohrenwend. B. S.\& Dohrenwend, B. P. (1981). Life stress and illness: Formulation of the issues. In B. S. Dohrenwend \& B. P. Dohrenwend (Eds.), Stressful life events and their contexts (pp. 1-27). New York: Neale Watson Academic Publications.

Ellenberger, H. F. (1970). The discovery of the unconscious. New York: Basic Books.

Engel, G. L. (1961). Is grief a disease? Psychosomatic Medicine, $\underline{23}, 18-22$. 
Engel. G. L. (1964). Grief and grieving. American Journal of Nursing, $\underline{64}(9), 93-98$.

English, R. W. (1971). The application of personality theory to explain psychological reaction to physical disability. Rehabilitation. Research and Practice Rev., $\underline{3}(1), 3 \overline{5-47}$.

Erikson, E. (1958). The problem of ego identity. Journal of the American Psychiatric Association, $\underline{4}, 56-121$.

Erikson, E. H. (1963). Childhood and Society (2nd ed.) New York: Norton (1st ed. 1950).

Erikson, E. H. (1968). Identity: Youth and crisis. New York: w. w. Norton.

Falek A., \& Britton S. (1974). Phases in coping: The hypothesis and its implications. Social Biology, 21, $1-7$.

Falvo, D. R. (1991). Medical and psychosocial aspects of chronic illness and disability. Gaithersburg, MD: Aspen Publishers, Inc.

Fine, M., \& Asch, A. (1981). Disabled women: Sexism without the pedestal. Journal of Sociology and Social Welfare, $\underline{8}, 233-248$.

Fink, S. L. (1967). Crisis and motivation: A theoretical model. Archives of Physical Medicine and Rehabilitation, $\underline{48}$, 592-597.

Fordyce, W. E. (1964). Personality characteristics in men with spinal cord injury as related to manner of onset of disability. Archives of Physical Medicine and Rehabilitation, 45, 321-325.

Frears, L. H., \& Schneider, J. M. (1981). Exploring loss and grief within a wholistic framework. The Personal and Guidance Journal, $\underline{59}(6), 341-346$.

Freud, S. (1953). Three essays on the theory of sexuality. In J. Strachey (Ed. and Trans.), The standard edition of the complete psychological works of Sigmund Freud (Vol. 7, pp. 125-245). London: Hogarth Press. (Original work published 1905) 
Freud, S. (1957). Mourning and melancholia. In J. Strachey (Ed. and Trans.), The standard edition of the complete psychological works of Sigmund Freud (Vol. 14, pp. 243-258). London: Hogarth Press. (Original work published 1917)

Frey, D. (1992). Psychological factors related to the recuperation of accident victims. In L. Montada, S. Flilipp, M. Lerner (Eds.), Life crises and experiences of loss in adulthood, (pp. 57-63). Hillsdale, NJ: Lawrence Erlbaum Associates, Inc.

Friedland, J., \& McColl, M. (1992). Disability and depression: Some etiological considerations. Social Science and Medicine, 34 (4), 395-403.

Garza, R. (1986). Socio-economic and cultural problems affecting delivery of rehabilitation services to Hispanic blind and visually disabled individuals Some observations. In S. Walker, F. Belgrave, A. Banner, \& S. Nicholls (Eds.), Equal to the challenge: Perspectives, problems and strategies in the rehabilitation of the non-White disabled (pp. 67-69). Washington, DC: The Bureau of Educational research, School of Education, Howard University.

Gilligan, C. (1982). In a different voice. Cambridge, MA: Harvard University Press.

Glueckauf, R. L., \& Quittner, A. L. (1984). Facing physical disabilities as a young adult: Psychological issues and approaches. In M. G. Eisenberg, L. Sutkin, \& M. Jansen (Eds.). Chronic illness and disability through the life span (pp. 167-183). New York: Springer Publishing Company.

Grossman, E. F. (1990). The Gestalt approach to people with amputations. Journal of Applied Rehabilitation Counseling, 21 (1), 16-19.

Grzesiak, R. C., \& Hicok, D. A. (1994). A brief history of psychotherapy and physical disability. American Journal of Psychotherapy, 48(2), 181-194.

Hainsworth, M. A., Eakes, G. G., Burke, M. L. (1994). Coping with chronic sorrow. Issues in Mental Health Nursing, 15, 59-66. 
Hanna, W. J., \& Rogovsky, E. (1991). Women with disabilities: Two handicaps plus. Disability, Handicap and Society, $\underline{6}(1), 49-62$.

Hanna, W. J., \& Rogovsky, E. (1992). On the situation of African-American women with physical disabilities. Journal of Rehabilitation, 60(1), 36-40.

Heikkinen, C. A. (1981). Loss resolution for growth. The Personal and Guidance Journal, $\underline{59}(6), 327-332$.

Hendrick, S. S. (1981). Spinal cord injury: A special kind of loss. Personal and Guidance Journal, $\underline{59}(6), 355-$ 359 .

Hoffman, A. D., Becker, R. D., \& Gabriel, H. P. (1976). The hospitalized adolescent: A guide to managing the ill and injured youth. New York: Free Press.

Horowitz, M. J. (1983). Psychological response to serious life events. In S. Breznitz (Ed.), The Denial of Stress, (pp. 129-159). New York: International Universities Press.

Hughes, F. (1980). Reactions to loss: Coping with disability and death. Rehabilitation Counseling Bulletin, 23(4), 251-257.

Jabaley, M. E., Hoopes, J. E., Knorr. N. J., \& Meyer, E. (1970). The burned child. In M. Debuskey (Ed.), The chronically ill child and his family (pp. 89-109). Springfield, IL: CC. Thomas.

Karl, G. T. (1987). A new look at grief. Journal of Advanced Nursing, $\underline{12}$, 641-645.

Keany, K. C., \& Glueckauf, R. L. (1993). Disability and value changes: An overview and analysis of acceptance of loss theory. Rehabilitation Psychology, 38(3), 199-210.

Kivnick, H. Q. (1985). Disability and psychosocial development in old age. Rehabilitation Counseling Bulletin, 29(2), 123-134.

Klass, D. (1981-82). Elisabeth Kuibler-Ross and the tradition of the private sphere: An analysis of symbols. Omega, 12(3), 241-267. 
Kowalsky, E. L. (1978) . Grief: A lost life-style. American Journal of Nursing, $\underline{78}, 418-420$.

Ribler-Ross, E. (1969) - On death and dying - New York: Macmillan.

Kübler-Ross, E. (1974). Questions and answers on death and dying. New York: Macmillan.

Kuibler-Ross, E. (1987). AIDS: The ultimate challenge. New York: Macmillan.

Langer, K. G. (1994). Depression and denial in psychotherapy of persons with disabilities. American Journal of Psychotherapy, $\underline{48}(2), 181-194$.

Lerner, M. J., \& Gignac A. M. (1992). Is it coping or is it growth?: A cognitive-affective model of contentment in the elderly. In L. Montada, S. Flilipp, \& M. Lerner (Eds.), Life crises and experiences of loss in adulthood (pp. 321-337). Hillsdale, NJ: Lawrence Erlbaum Associates.

Lifton, R. J., 1975. The broken connection. New York: Simon and Schuster.

Lilliston, B. A. (1985). Psychosocial responses to traumatic physical disability. Social Work in Health Care, $10(4), 1-13$.

Lindemann, E. (1944). Symptomatology and management of acute grief. American Journal of Psychiatry, 101, $141-148$.

Lindgren, C. L., Burke, M. L., Hainsworth, M. A., \& Eakes, G. G. (1992) - Chronic sorrow: A lifespan concept. Scholarly Inquiry for Nursing Practice, $\underline{6}(1), 27-40$.

Linkowski, D. C. (1971). The acceptance of disability scale. (Available from Dr. Linkowski, George Washington University, Washington, DC).

Lorenz, K. Z . (1935). Der Kumpan in der Umwelt des Vogels (The companion in the bird's world). Journal fuer ornithologie, 83, 137-213. (Abbreviated English translation published 1937 in Auk, 54, 245-273). 
Maddux, J. E., Roberts, M. C., Sledden, E. A., \& Wright, L. (1986). Developmental issues in child health psychology - American Psychologist, 41(1), 25-34.

Marshak, L. E., \& Seligman, M. (1993). Counseling persons, with physical disabilities: Theoretical and clinical perspectives. Austin, TX: Pro-Ed.

McDaniel, J. W. (1969). Physical disability and human behavior. New York: Pergamon Press.

MCWhirter, J. J., \& MCWhirter, B. T. (1991). A framework for theories in counseling. In D. Capuzzi, \& D. Gross (Eds.), Introduction to counseling: Perspectives for the 1990s (pp. 69-88). Boston: Allyn and Bacon.

Meyerson, L. (1971). Somatopsychology of physical disability. In w. Cruickshank (Ed.), Psychology of exceptional children and youth (2nd ed., pp. 1-71). Englewood Cliffs, NJ: Prentice-Hall.

Monte, C. F. (1987). Beneath the mask: An introduction to theories of personality (3rd ed.). New York: Holt, Rinehart and winston.

Moos, R. H., \& Tsu, V. D. (1977). The crisis of physical illness: An overview. In R. Moos (Ed.), Coping with physical illness (pp. 3-21). New York: Plenum Medical Book Co.

Morris, w. (Ed.). (1980). The American heritage dictionary of the English language (College ed.). Boston: Houghton Mifflin.

Neugarten, B. (Ed.). (1968). Middle age and aging. Chicago: University of Chicago Press.

Neugarten, B. L. (1977). Personality of aging. In J. Birren \& K. Schaie (Eds.), Handbook of the psychology of aging (pp. 626-644). New York: Van Nostrand Reinhold.

Nolen-Hoeksema, S. (1987). Sex differences in unipolar depression: Evidence and theory. Psychological Bulletin, 101, 259-282.

Nye, R. D., (1986). Three psychologies: perspectives from Freud, Skinner, and Rogers. Pacific Grove, CA: Brooks/Cole. 
Olshansky, S, (1962). Chronic sorrow: A response to having a mentally defective child. Social Casework, 43, 191193.

Osterweis, M., Solomon, F., \& Green, M. (Eds.), (1984). Bereavement: Reactions, consequences, and care. Washington, DC: National Academy Press.

Papanek, H. (1967). Cultural and interpersonal psychoanalytic theories. In A. Freedman, H. I. Kaplan, \& H. S. Kaplan (Eds.), Comprehensive textbook of psychiatry (pp. 320-326). Baltimore, MD: The Williams \& Wilkins.

Parkes, C. M. (1970a). Seeking and finding a lost object: Evidence from recent studies of the reaction to bereavement. Social Science and Medicine, $\underline{4}, 187-201$.

Parkes, C. M. $(1970 \mathrm{~b})$. The first year of bereavement. Psychiatry, 33, 444-467.

Parkes, C. M. (1972). Bereavement: Studies of grief in adult life. New York: International Universities Press.

Parkes, C. M. (1985). Bereavement. British Journal of Psychiatry, 146, 11-17.

Parry, J. K. (1994). Death review: An important component of grief resolution. Social Work in Health Care, 20 (2), 97-107.

Peretz, D. (1970a). Development, object-relationships, and loss. In B. Schoenberg, A. Carr. D. Peretz, \& A. Kutscher (Eds.), Loss and grief: Psychological management in medical practice (pp. 3-19). New York: Columbia University Press.

Peretz, D. (1970b). Reaction to loss. In B. Schoenberg, A. Carr. D. Peretz, \& A. Kutscher (Eds.), Loss and grief: Psychological management in medical practice (pp. 21-35) • New York: Columbia University Press.

Piaget, J. (1950). The psychology of intelligence. New York: Harcourt Brace \& World. (1st ed. 1936)

Piaget, J. (1954). The construction of reality in the child. New York: Basic Books. (1st ed. 1937) 
Piaget, J. (1963). The origins of intelligence in children. New York: Norton. (1st ed. 1952)

Piaget, J. (1965). The moral judgment of the child. New York: The Free Press. (1st ed. 1932)

Power, P. W., Hershenson, D. B., \& Schlossberg, N. K. (1985). Midlife transition and disability. Rehabilitation Counseling Bulletin, 29(2), 100-111.

Pulver, S. (1970). Narcissism: The term and the concept. Journal of the American Psychoanalytic Association, $18,319-341$.

Reich, J. W., Zautra, A. J., \& Guarnaccia, C. A. (1989). Effects of disability and bereavement on the mental health and recovery of older adults. Psychology and Aging, $4(1), 57-65$.

Rodgers B. L., \& Cowles, K. V. (1991). The concept of grief: An analysis of classical and contemporary thought. Death Studies, 15, 443-458.

Rokeach, M. (1973). The nature of values. New York: Free Press.

Rule, W. R. (1985). An Adlerian perspective. Journal of Applied Rehabilitation Counseling, 16(3), 9-14.

Russell, R. A. (1981). Concepts of adjustment to disability: An overview. Rehabilitation Literature, $\underline{42}, 330-338$.

Rustad, L. C. (1984). Family adjustment to chronic illness and disability in mid-life. In M. G. Eisenberg, L. Sutkin, \& M. Jansen (Eds.). Chronic illness and disability through the life span (pp. 222-242). New York: Springer Publishing Company.

Schneider, J. M. (1980). Clinically significant differences between grief, pathological grief, and depression. Patient Counseling and Health Education, vol. 4, 161-169.

Schulz, R., \& Aderman, D. (1974). Clinical research and the stages of dying. Omega, $\underline{5}(2), 137-143$.

Selye, H. (1956). Stress of life. New York: MCGraw-Hill. 
Shadish, W. R., Hickman, D., \& Arrick, M. C. (1981) . Psychological problems of spinal cord injury patients: Emotional distress as a function of time and locus of control. Journal of Counseling and Clinical Psychology, $\underline{49}, 297$.

Shilling, L. E. (1984). Perspectives on counseling theories. Englewood Cliffs, NJ: Prentice-Hall.

Shontz, F. C. (1973). Severe chronic illness. In J. Garrett \& E. Levine (Eds.), Rehabilitation practices with the physically disabled (pp. 119-148). New York: Columbia University Press.

Shontz, F. C. (1977). Six principles relating disability and psychological adjustment. Rehabilitation Psychology, 24 (4), 207-210.

Shontz, F. C. (1978). Psychological adjustment to physical disability: Trends in theories. Archives of Physical Medicine and Rehabilitation, 59, 251-254.

Shontz, F. C., 1980. Theories about the adjustment to having a disability. In W. Cruickshank (Ed.), Psychology of exceptional children and youth (4th ed.), (pp. 3-43). Englewood Cliffs, NJ: Prentice Hal1.

Sigelman, C. K., \& Shaffer, D. R. (1991). Life-span human development. Pacific Grove, CA: Brooks/Cole Publishing Company.

Silver, R. C. (1982). Coping with an undesirable life event: A study of early reactions to physical disability. Unpublished doctoral dissertation, Northwestern University, Evanston, IL.

Silver, R. L., \& Wortman, C. B. (1980). Coping with undesirable life events. In J. Garber \& M. E. Seligman (Eds.), Human helplessness: Theory and applications (279-340) New York: Academic Press.

Smart, J. F., \& Smart, D. W. (1991). Acceptance of disability and the Mexican American culture. Rehabilitation Counseling Bulletin, 34 (4), 357-367.

Smart, J. F., \& Smart, D. W. (1994). The rehabilitation of Hispanics experiencing acculturation stress: Implications for practice. Journal of Rehabilitation, $\underline{60}(4), 8-12$. 
Smith, J. H. (1975). On the work of mourning. In B. Schoenberg, I. Gerber, A. Wiener, A. Kutscher, D. Peretz, A. Carr (Eds.), Bereavement: Its psychosocial aspects (pp. 19-49). New York: Columbia University Press.

Sodowsky, G. R., Maguire, K., Johnson, P., Ngumba, W., \& Kohles, R. (1994). World views of White-American, Mainland Chinese, Taiwanese, and African students. Journal of Cross-Cultural Psychology, 25(3), 309-324.

Stewart, J. R. (1994). Denial of disabling conditions and specific interventions in the rehabilitation counseling setting. Journal of Applied Rehabilitation Counseling, $25(3), 7-15$.

Stewart, T., \& Shields, C. R. (1985). Grief in chronic illness: Assessment and management. Archives of Physical Medicine and Rehabilitation, 66, 447-450.

Stephenson, J. S., \& Murphy, D. (1986) . Existential grief: The special case of the chronically ill and disabled. Death Studies, 10, 135-145.

Stroebe, M. S. (1992-93). Coping with bereavement: A review of the grief work hypothesis. Omega, 26(1), $19-42$.

Stroebe, W., \& Stroebe, M. S. (1993). Determinants of adjustment to bereavement in younger widows and widowers. In M. Stroebe, R. Hansson, \& W. Stroebe (Eds.), Handbook of bereavement: Theory, research, and intervention (pp. 208-226). New York: Cambridge University Press.

Sutkin, L. C. (1984) . Introduction. In M. G. Eisenberg, L. Sutkin, \& M. Jansen (Eds.). Chronic illness and disability through the life span (pp. 1-19). New York: Springer.

Taylor, S. E. (1983). Adjustment to threatening events: A theory of cognitive adaptation. American Psychologist, 38, 1161-1173.

Teel, C. (1991). Chronic Sorrow: Analysis of the concept. Journal of Advanced Nursing, 16, 1311-1319.

Thurer, S. (1985). Rehabilitation counseling: A psychodynamic perspective. Journal of Applied Rehabilitation Counseling, $1 \overline{6}(3), 4-8$. 
Trieschmann, R. B. (1988). Spinal cord injuries: Psychological, social, and vocational rehabilitation. New York: Demos.

Turner, R. J., \& Noh, S., (1988). Physical disability and depression: A longitudinal analysis. Journal of Health and Social Behavior, 29, 23-37.

U. S. Bureau of the Census, (1989). Labor force status and other characteristics of persons with a work disability. 1981 to 1988. (Current Population Reports Series P-23 No 160). Washington: U.S. Government Printing Office.

Vash, C. L. (1981). The psychology of disability. New York: Springer.

Vines, D. W. (1986). Regrieving in parents of disabled adolescents. (Doctoral dissertation, Boston University, 1986). Dissertation Abstracts International, 46, 387A.

Weenolsen, P. (1988). Transcendence of loss over the life span. New York: Hemisphere.

Whitt, J. K. (1984). Children's adaptation to chronic illness and handicapping conditions. In M. Eisenberg, L. Sutkin, \& M. Jansen (Eds.). Chronic illness and disability through the life span (pp. 69-102). New York: Springer Publishing.

Wiker, L., Wasow, M., \& Hatfield, E. (1981). Chronic sorrow revisited: Parent vs. professional depiction of the adjustment of parents of mentally retarded children. American Journal of Orthopsychiatry, 51, 63-70.

Wong, P. T., \& Weiner, B. (1981). When people ask "why" questions, and the heuristics of attributional search. Journal of Personality and Social Psychology, 40, 650663.

Wortman, C. B., \& Silver, R. C. (1987). Coping with irrevocable loss. In G. VandenBos, \& B. Bryant, (Eds.), Cataclysms, crises, and catastrophes: Psychology in action. The master lectures, (Vol. 6, pp. 189-235). Washington, DC: American Psychological Association. 
Wortman, C. B., \& Silver, R. C. (1989). The myths of coping with loss. Journal of Clinical and Consulting Psychology, 57, 349-357.

Wortman, C. B., \& Silver, R. C. (1992). Reconsidering assumptions about coping with loss: An overview of current research. In L. Montada, S. Flilipp, M. Lerner (Eds.), Life crises and experiences of loss in adulthood, (pp. 341-365). Hillsdale, NJ: Lawrence Erlbaum.

Wright, B. A. (1983). Physical disability: A psychosocial approach (2nd ed.). New York: Harper and Row.

Zisook, S, \& DeVaul, R. (1985). Unresolved grief. The American Journal of Psychoanalysis, $45(4), 370-379$.

Zola, I. K. (1982). Missing pieces: A chronicle of living with a disability. Philadelphia: Temple Univeristy Press. 